\title{
Un foyer du Néolithique moyen à Luzéronde (Noirmoutier-en-l'île, Vendée)
}

A middle neolithic fireplace at Luzéronde (Noirmoutier-en-l'île, Vendée)

Eine mittelneolithische Feuerstelle bei Luzéronde (Noirmoutier-en-l'île, Vendée)

Un hogar del Neolítico medio en Luzéronde (Noirmoutier-en-l'île, Vendée)

Jean-Marc Large et Thomas Vigneau

\section{CpenEdition}

\section{Édition électronique}

URL : https://journals.openedition.org/rao/6753

DOI : 10.4000/rao.6753

ISSN : $1775-3732$

Éditeur

Presses universitaires de Rennes

\section{Édition imprimée}

Date de publication : 21 décembre 2021

ISBN : 978-2-7535-8668-0

ISSN : 0767-709X

\section{Référence électronique}

Jean-Marc Large et Thomas Vigneau, « Un foyer du Néolithique moyen à Luzéronde (Noirmoutier-enl'île, Vendée) », Revue archéologique de l'Ouest [En ligne], 37 | 2021, mis en ligne le 21 décembre 2021 consulté le 10 octobre 2022. URL : http://journals.openedition.org/rao/6753 ; DOI : https://doi.org/ $10.4000 /$ rao. 6753 


\title{
Un foyer du Néolithique moyen à Luzéronde (Noirmoutier-en-l'île, Vendée)
}

\author{
A middle neolithic fireplace at Luzéronde (Noirmoutier-en-l'île, Vendée)
}

\author{
Jean-Marc Large \\ Chercheur associé IMR 6566 CReAHH, Rennes 1 (jlarge2@wanadoo.fr) \\ Thomas Vigneau \\ Archéologue-topographe au département de la vendée (thomas.vigneau@vendee.fr)
}

\begin{abstract}
Résumé: Alors que la mer continue son travail de sape sur le littoral, quelques blocs brûlés sont apparus en coupe de falaise sur la pointe de Luzéronde à Noirmoutier. Le site était déjà connu et une intervention archéologique a eu lieu en 1986. La structure de combustion qui apparaissait ne faisait pas partie du secteur d'intervention ancien. Devant sa menace de destruction définitive, une intervention très limitée a eu lieu en octobre 2019 et a permis de mieux circonscrire la structure associée à un gros bloc de gneiss. Une datation au radiocarbone la positionne dans le troisième quart du $\mathrm{V}^{e}$ millénaire av. n. è.
\end{abstract}

Abstract: As the sea continues its work of undermining the coastline, a few burnt blocks have appeared in cliff sections on the Pointe de Luzéronde in Noirmoutier. This is a known site and an archaeological intervention took place there in 1986, the burnt feature that appeared here was not part of the excavation. Faced with the threat of its destruction, an intervention took place in October 2019 in order to better circumscribe the feature and an associated element. A radiocarbon dating places it in the third quarter of the 5 th millennium BC.

Mots clés: Pays de la Loire, Vendée, Néolithique, foyer.

Keywords: Pays de Loire, Vendée, Neolithic, hearth.

De par son substrat en roche métamorphique et en dépôts éocènes, la partie nord de l'île de Noirmoutier a été le support de nombreuses implantations néolithiques. Les sites mégalithiques y étaient très nombreux avant leur destruction massive au XIX ${ }^{\mathrm{e}}$ siècle. Par contre, les sites d'occupations domestiques sont bien moins connus sur l'île et celui de Luzéronde, occupé dès le Néolithique ancien, voire même au Mésolithique, a connu une longue présence jusqu'à l'âge du Bronze (fig. 1). Le site a malheureusement subi une modification drastique lors de la dernière guerre : les Allemands ont installé des abris souterrains et des postes pour mitrailleuses (Chazette, 2010). Malgré cette importante dégradation, il reste des parties non touchées qui font l'objet d'une érosion naturelle par la mer. C'est à cette occasion qu'une petite structure de combustion a été repérée sur la microfalaise de la pointe de Luzéronde et a fait l'objet d'une intervention avant sa destruction par les éléments naturels.

\section{HISTORIQUE}

\section{Les premières prospections archéologiques et le sondage urgent (1986)}

Lorsque Viviane Borne en 1986, alors en thèse de géologie à l'université de Nantes, signala aux Antiquités préhistoriques de l'époque la présence de nombreux éléments en silex et en poterie sur une bande de $100 \mathrm{~m}$ qui longeait la côte, un sauvetage urgent fut décidé, dirigé par F. Letterlé alors au service des Antiquités de Nantes (fig. 2). Il a eu lieu en mai 1986 sur une avancée de la falaise qui subissait les assauts de la mer lors des tempêtes hivernales. La surface fouillée était d'une dizaine de mètres carrés. La principale couche dite « de terre sableuse gris-brun, reposant directement sur le socle granitique (sic) " a été fouillée par passes d'une dizaine de centimètres. À cet endroit, l'épaisseur de 
ladite couche était comprise entre 15 et $35 \mathrm{~cm}$ et elle disparaissait sur les surfaces les plus érodées.

Aucune structure n'a été repérée sur cette surface fouillée, aucune concentration non plus de mobilier; il n'a pas été possible de déterminer une occupation claire et franche alors que la couche recélait un nombre important de mobilier : 1892 pièces lithiques (189 pièces au mètre carré en moyenne) et environ 500 tessons (50 tessons au mètre carré). L'ensemble du mobilier recueilli, tant céramique que lithique, fait référence essentiellement au Bronze ancien avec un bruit de fond mésolithique (fig. 3). L'intention de l'époque était claire : il s'agissait de documenter un site menacé par l'érosion marine et les passages répétés de promeneurs. La présence de mobilier du Bronze ancien dans une couche sans doute très remaniée permettait alors d'envisager une possible suite à l'étude de ce site.

\section{Les prospections ultérieures (2007-2019)}

La surveillance de ce site a repris dans les années 2000 sous l'égide du Groupe Vendéen d'Études Préhistoriques (GVEP) qui organisa plusieurs campagnes de prospections le long du littoral vendéen et, en particulier, à Noirmoutier. En 2009, la découverte des premiers tessons du Néolithique ancien relança l'intérêt pour ce site qui ne pouvait plus être dédié seulement au Bronze ancien. Les tessons retrouvés indiquaient une présence du Néolithique ancien centre-Atlantique, d'affinité méridionale, avec la présence de décors à la coquille (fig. 4, 1-2). Luzéronde devenait ainsi le site le plus septentrional de ce groupe culturel, au contact avec l'influence VSG (Large, 2009). Ce constat est très important car il va induire la recherche dans les années qui suivirent. De par la présence constante
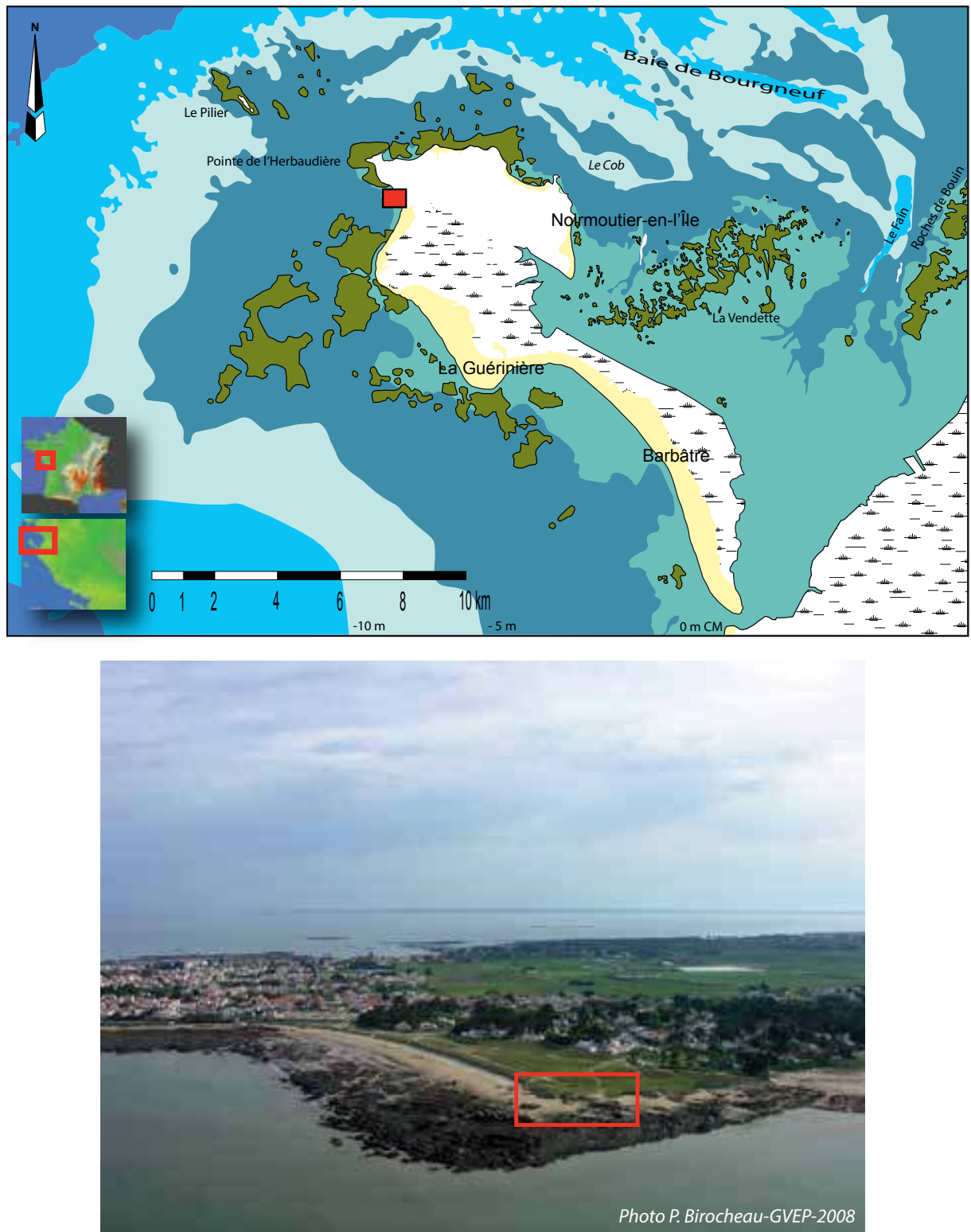

Figure 1 : Situation du site de la pointe de Luzéronde à Noirmoutieren-l'île (DAO J.-M. Large, cliché P. Birocheau).

Figure 1: Location of the Pointe de Luzéronde site at Noirmoutieren-l'île (CAD J.-M. Large, photo P. Birocheau). 
sur l'île de Noirmoutier d'un membre du GVEP, le site allait pouvoir être surveillé de manière continue afin d'évaluer l'érosion côtière qui ne manquerait pas de mettre à jour des structures inédites.

Dès 2011, il fut remarqué des pierres ayant subi une combustion qui ressortaient de la coupe de falaise au nord-ouest de la fouille de 1986, toujours dans ce niveau "de terre sableuse gris-brun » fouillé par F. Letterlé qui a été nommé par la suite "sables limoneux " (2009). Le mobilier repéré lui aussi en coupe de falaise était très hétérogène. Aux présences au Néolithique ancien et au Bronze ancien, il fallait y ajouter le Néolithique récent
(Groh-Collé) grâce à des tessons de céramique ainsi que des armatures (fig. 4, 4-13). En 2017, le nombre de pierres brûlées apparues en coupe de falaise, toujours dans le même secteur, augmentait et plusieurs de ces pierres étaient quasiment jointes, reposant à l'horizontale : une structure à pierres chauffées apparaissait dans un secteur de la côte partiellement protégé par un relief de la roche exposé aux vents dominants et aux coups de boutoir de l'océan. Pendant deux ans, cette structure va faire l'objet d'une attention soutenue jusqu'à ce que les premières pierres disparaissent de la coupe en raison de l'érosion du trait de côte.

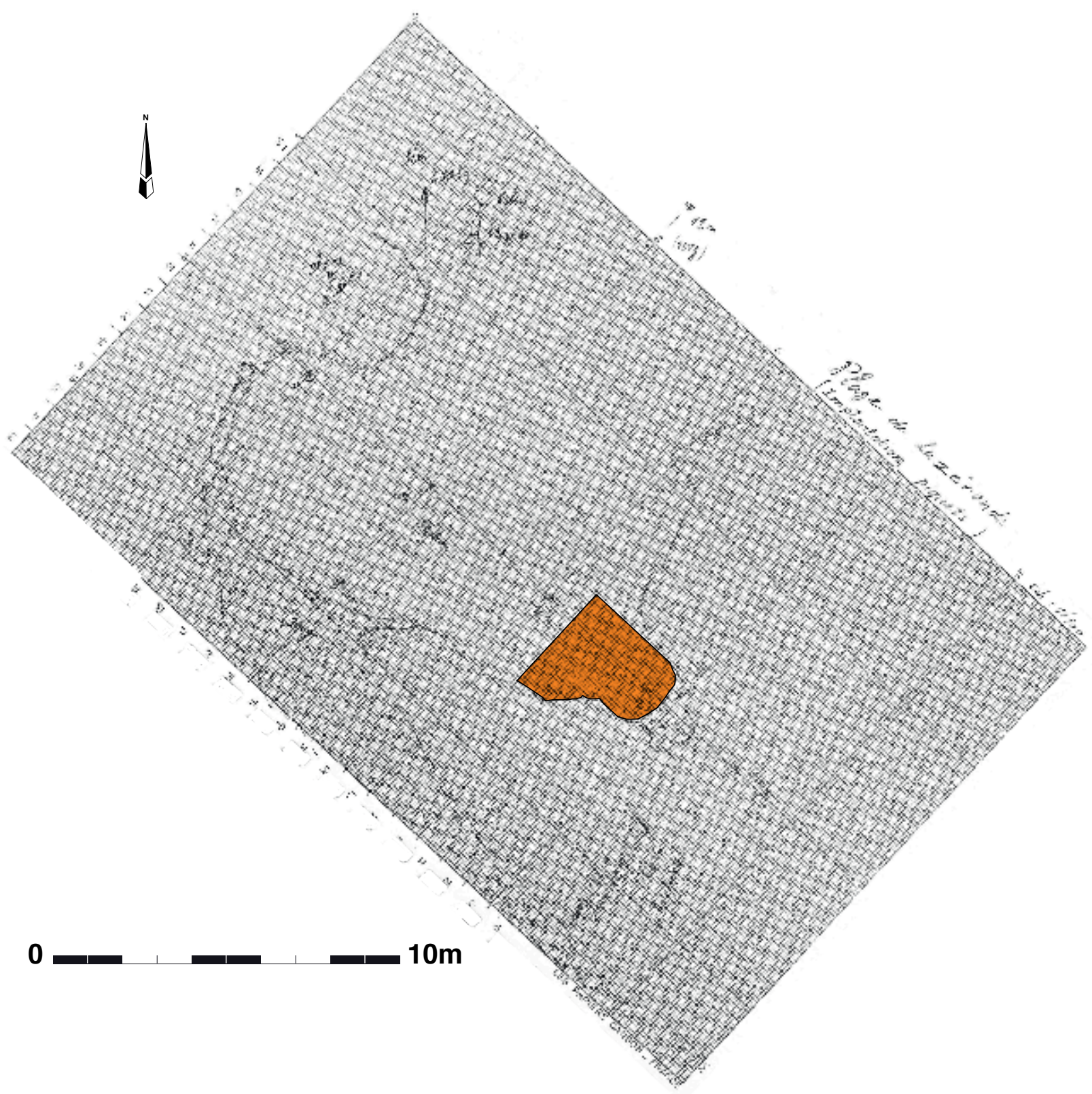

Figure 2 : Situation de l'intervention de sauvetage de F. Letterlé en 1986 sur la pointe de Luzéronde et coupe relevée lors de la fouille (relevés F. Letterlé).

Figure 2: Location of the salvage operation carried out by F. Letterlé in 1986 on the Pointe de Luzéronde site and cross-section taken during the excavation (drawings by F. Letterlé). 

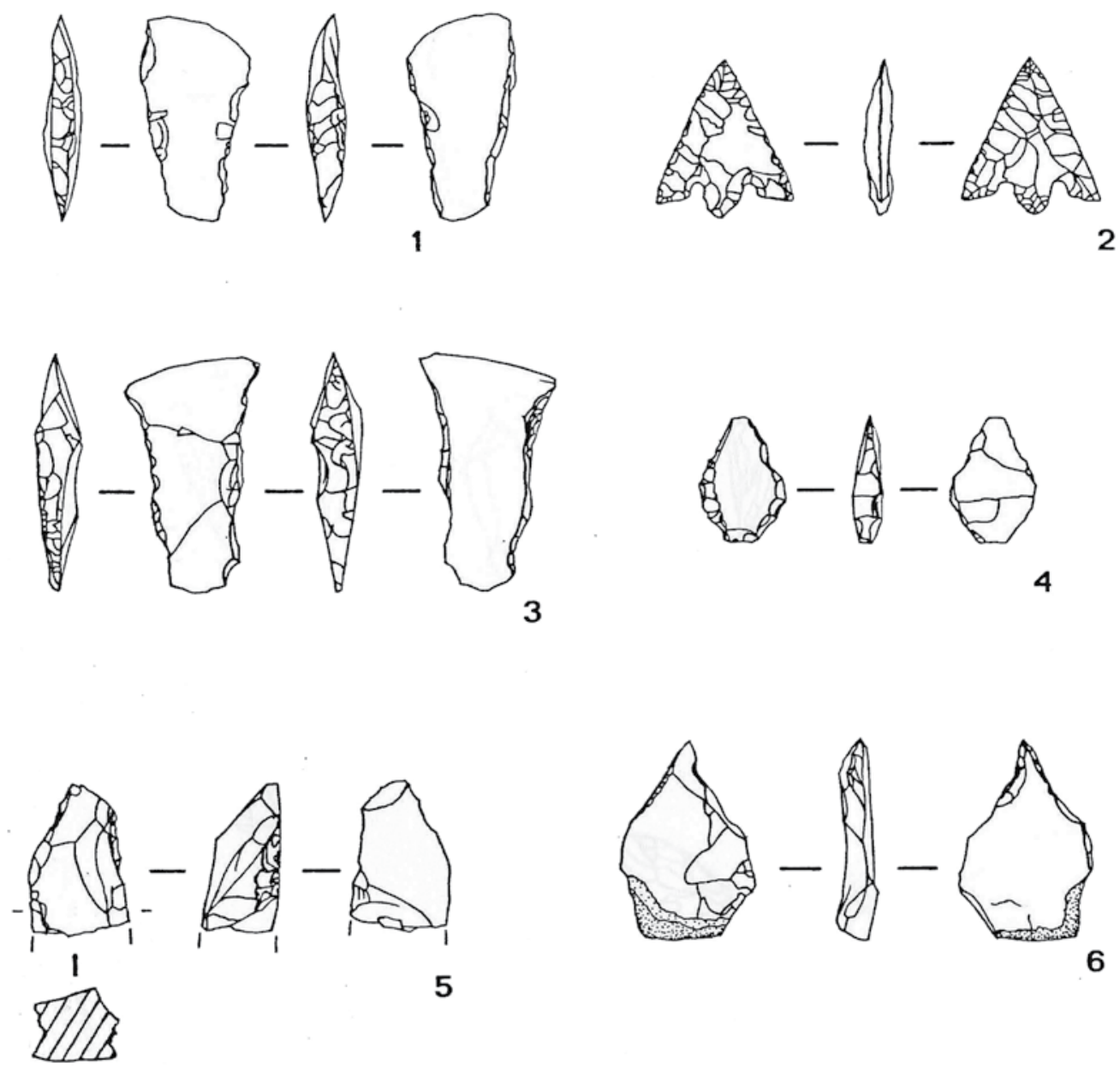

5

6
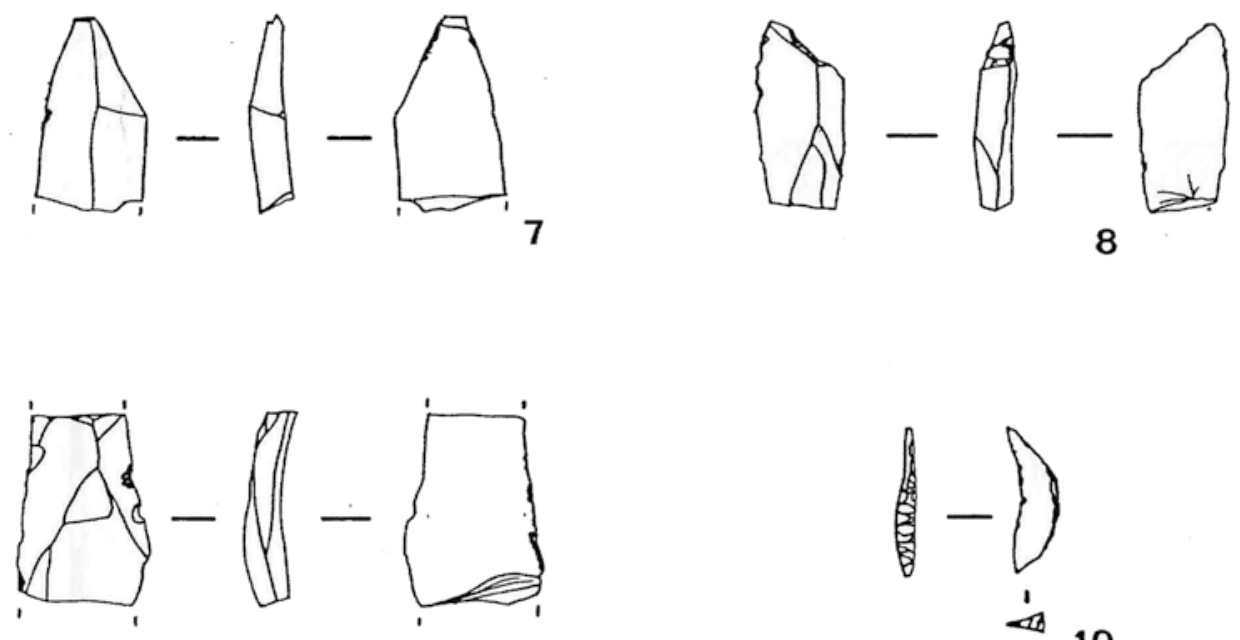

9

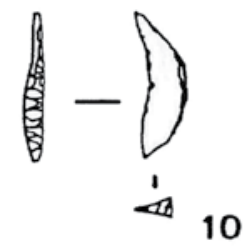

Figure 3 : Mobilier significatif retrouvé lors de l'intervention de F. Letterlé en 1986.

Figure 3: Significant finds brought to light during the intervention of F. Letterlé in 1986.

On remarque la présence d'une armature perçante à ailerons équarri (Bronze ancien), d’armatures tranchantes trapézoïdales à retouches abruptes (Néolithique récent), de poteries décorées à la cordelette et au cordon digité (Bronze ancien et moyen) [dessins F. Letterlé]. 

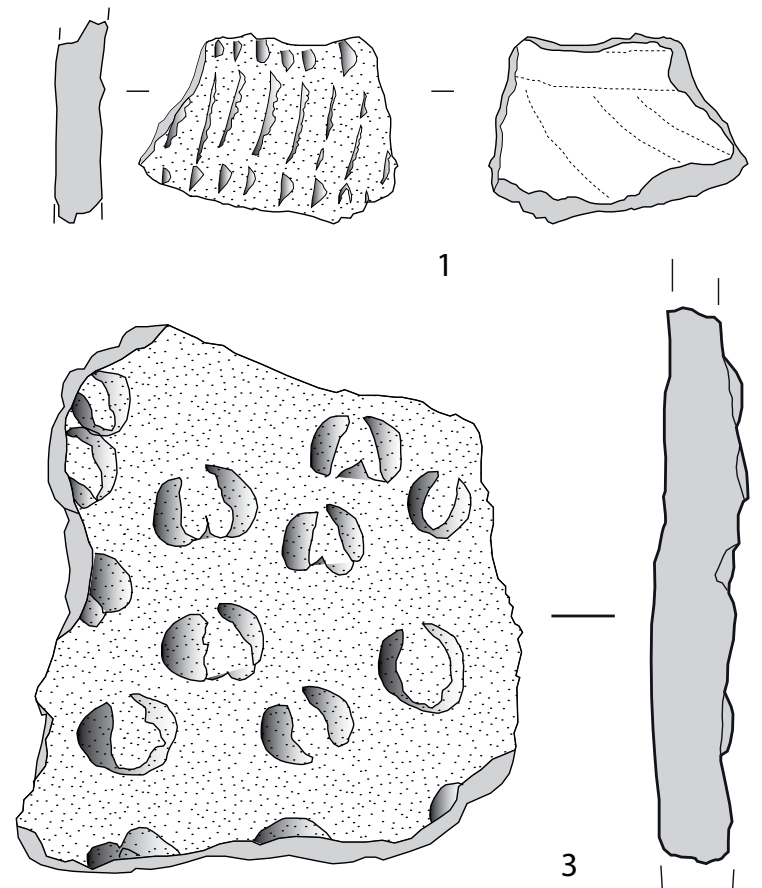

3

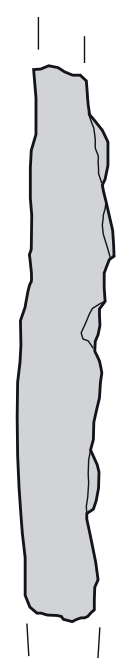

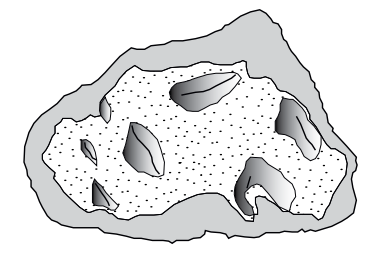

2
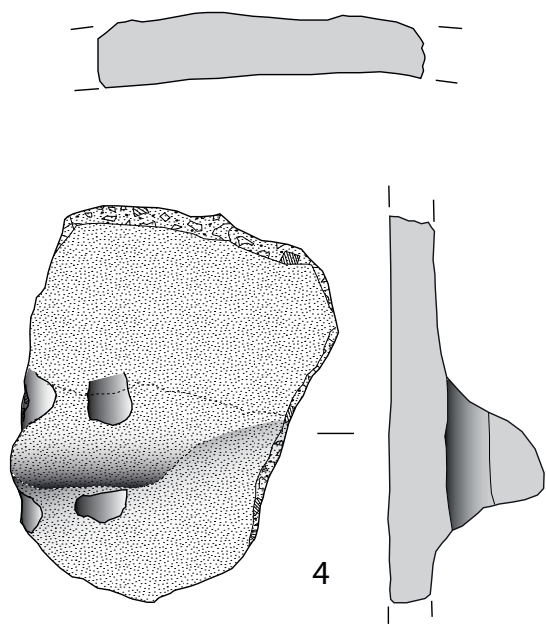

$0 \longrightarrow-5 \mathrm{~cm}$
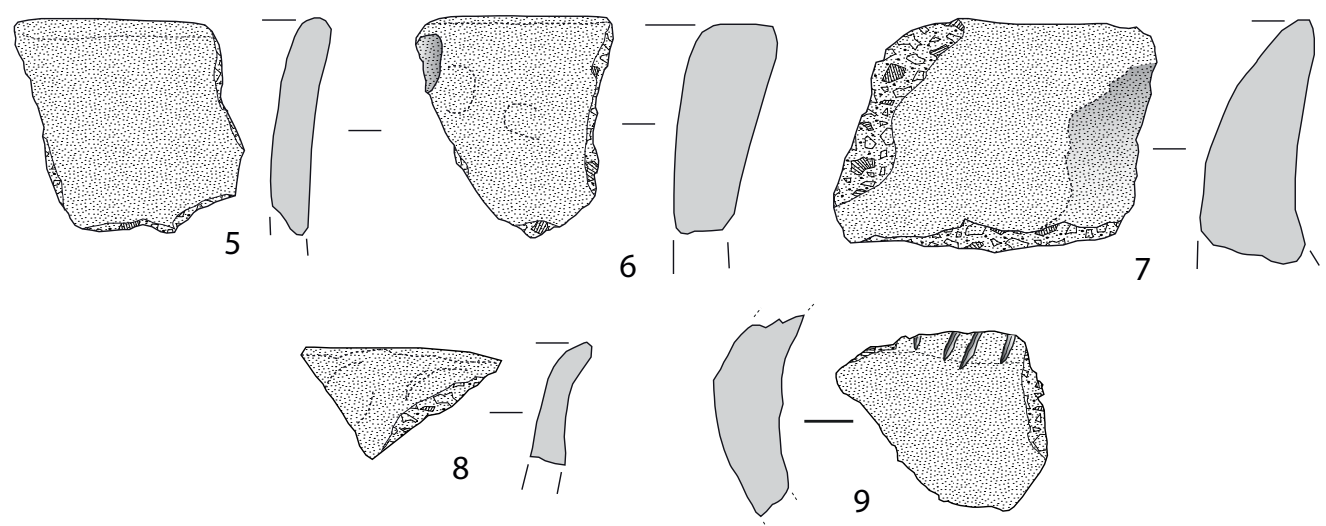

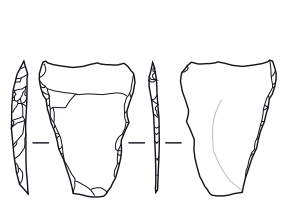

10

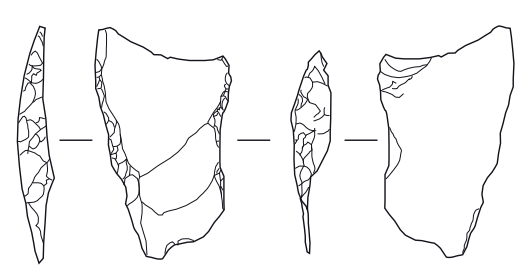

11

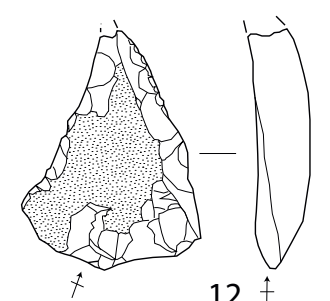

$12 \uparrow$

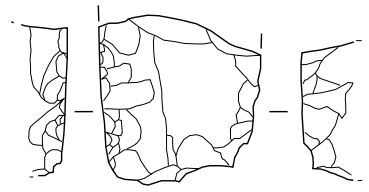

13

Figure 4 : Mobilier trouvé en prospection pédestre lors de la surveillance du site.

Figure 4: Fieldwalking finds at the site.

1-2 : tessons témoignant d'une influence méridionale rattachée au NACA, découverts en coupe de falaise en 2009 (Large, 2009) ; 3 : un autre tesson du Néolithique ancien fut découvert toujours en coupe de falaise en $2018 ; 4-13$ : d'autres tessons et mobilier lithique issus de ramassages de surface donnent des indications chronologiques variant du Néolithique moyen (armature $n^{\circ}$ 10) au Néolithique récent (tesson Groh-Collé $n^{\circ}$ 9) et au Bronze ancien (DA0 et dessin J.-M. Large).

1-2: sherds showing a southern influence attached to the NACA discovered in a cliff section in 2009 (Large, 2009); 3: sherd from the Early Neolithic discovered in a cliff section in 2018; 4-13: other sherds and lithics found on the surface date from the Middle Neolithic (armature no.10) to the Late Neolithic (Groh-Collé sherd no.9) and the Early Bronze Age (CAD and drawing J.-M. Large). 
Puis, en avril 2018, c'est un autre tesson à décor pincé, au doigt faisant référence lui aussi au Néolithique ancien, qui fut découvert. Sa présence à environ 1,20 m au nord des pierres brûlées laissait penser qu'un lien chronologique existait entre eux (fig. 4, 3). Les comparaisons pour ce type de céramique sont à la fois peu nombreuses et intéressantes. Le décor pincé au doigt est présent sur le site NACA de la pointe du Grouin du Cou à La Tranchesur-Mer (Joussaume, 1981) sur plusieurs tessons. Plus au sud, la référence incontournable est la grotte de Bellefonds (Patte, 1971) où plusieurs céramiques globulaires à paroi resserrée présentent un registre décoratif similaire à celui de Luzéronde. Le parallèle décoratif a aussi été fait avec des tessons de la grotte de Bois-Bertaud à Saint-Léger-les-Pons (Gachina et al., 1991), ainsi que dans la grotte de la Grande Roche de la Plématrie à Quinçay (Lévêque et al., 1997).
C'est aussi le cas pour le site des Ouchettes à Plassay où le registre décoratif des céramiques est très proche de celui des tessons de Luzéronde (Laporte et Picq 2002). Enfin, le site de Geay présente lui aussi des éléments comparables (Laporte, 2003). Toutefois, ces registres décoratifs au doigt sont aussi présents dans des contextes plus septentrionaux (à Betton par exemple, Blanchet et al., 2010) mais aussi plus continentaux (à Aubevoye, par exemple, Riche et al., 2010 ou à Sours, Dupont et al., 2010) dans des contextes rattachés au Villeneuve-Saint-Germain.

Du fait de la menace concernant la structure à pierres brûlées, il fut donc envisagé en mars 2019 de fouiller les restes de cette structure sur une surface très réduite afin de ne pas dénaturer et fragiliser la falaise (fig. 5). Cette intervention fut reportée à la fin octobre 2019, avant les tempêtes hivernales.
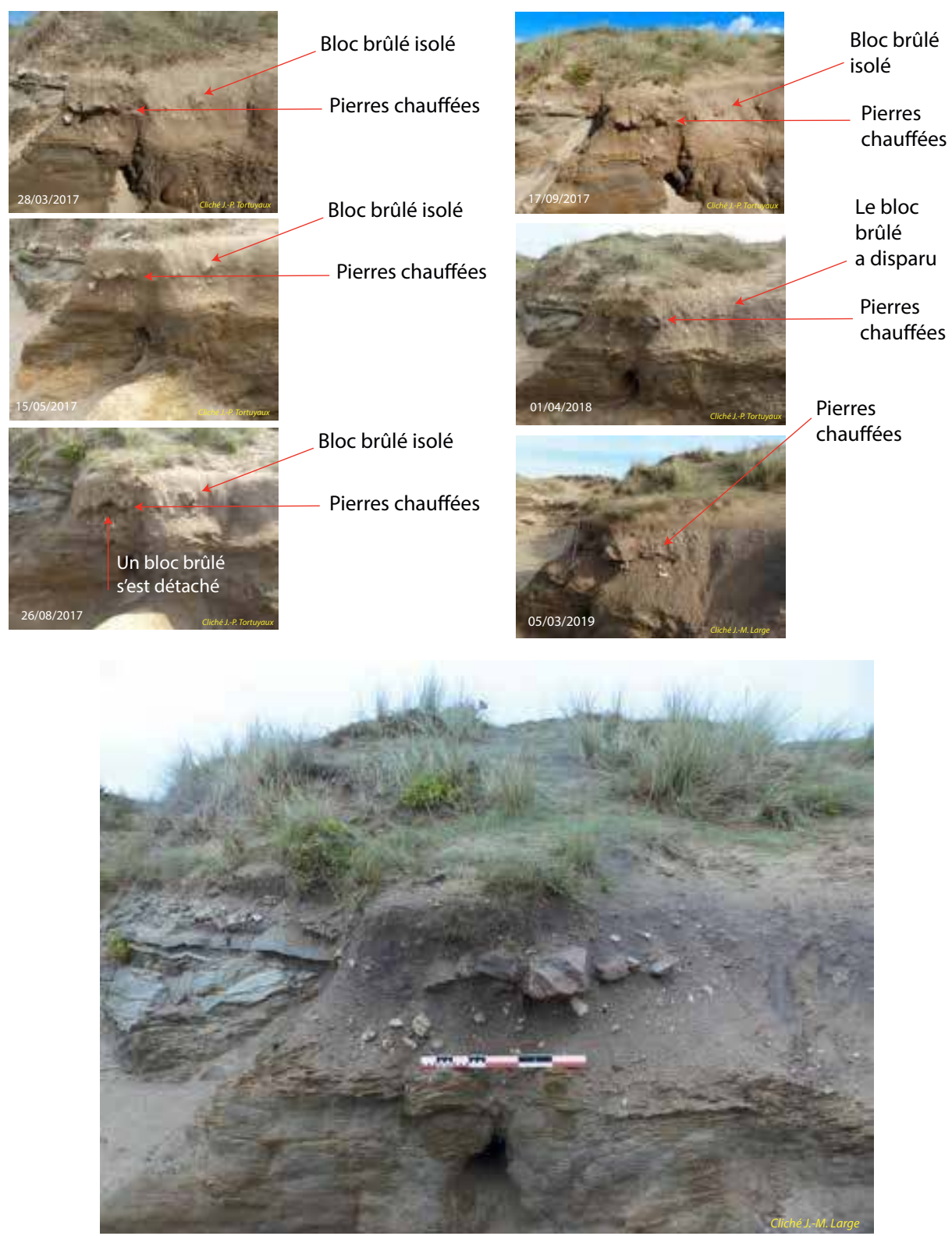

Figure 5 : En 2017, une série continue de blocs brûlés apparaît en coupe de falaise. Son observation attentive dans les mois qui suivent permet de se rendre compte de son état de dégradation; une intervention était nécessaire... (DAO et clichés J.-M. Large, J.P. Tortuyaux). En bas, état de la structure à pierres chauffées avant l'intervention (clichés du 27/10/2019, J.-M. Large).

Figure 5: A series of burnt blocks appeared in the cliff section 2017. Careful observation in the months that followed revealed its poor state of preservation, an intervention was deemed necessary... (CAD and photos by J.-M. Large, J.P. Tortuyaux). Below, state of the fire feature before the intervention (photos taken on 27/10/2019, J.-M. Large). 


\section{L'INTERVENTION D'OCTOBRE 2019}

La surface de l'emprise était faible : $4 \mathrm{~m}^{2}$ au maximum. Il s'agissait de documenter la structure à pierres chauffées qui apparaissait en coupe de falaise dans les niveaux sablo-limoneux (fig. 5). Une fois la pellicule sableuse de la dune récente enlevée (US1), peu épaisse en cet endroit (30 $\mathrm{cm}$ au maximum), le plafond de la couche sablo-limoneuse est apparu (US2). D'une épaisseur de 40 à $45 \mathrm{~cm}$, elle surmonte un autre niveau de limon sablo-argileux à graves siliceuses de 20 à $30 \mathrm{~cm}$ d'épaisseur (US3). C'est au contact de ces deux US que la structure à pierres chauffées était située, positionnée sur le sol formé par le plafond de l'US3. Le substrat gneissique apparaît ensuite, altéré au contact avec l'US3 et d'une topographie horizontale irrégulière. Une fois le plafond de l'US2 mis en évidence, une série de 10 passes manuelles successives a été effectuée (soit une passe de 4 à $5 \mathrm{~cm}$ d'épaisseur) pour atteindre la structure à pierres chauffées. L'ensemble du mobilier a été positionné dans l'espace pour l'ensemble des 10 passes.

\section{Le substrat géologique}

La partie nord de l'île de Noirmoutier est composée d'un noyau cristallin formé de gneiss, à l'ouest, et de granite, au centre et à l'est. Dans la partie sud de ce noyau, au contact avec les marais salants, le bloc cristallin est abrupt.
Le calcaire éocène, actuellement noyé par la transgression flandrienne, est en contact avec l'abrupt du noyau cristallin, passant très près de la pointe de Luzéronde dans une direction classique du réseau faillé du Massif armoricain (fig. 6).

La pointe nord-ouest de l'île de Noirmoutier est constituée par des gneiss métatectiques, traversés par d'innombrables filons de granite et de pegmatite. À la pointe de Luzéronde, affleurent des métatexites plissotées, hétérogènes, à structure grenue ou feuilletée, traversées par des masses décamétriques de granite fin clair et de pegmatite à muscovite incluant de minces cloisons de gneiss fin grenu à grenat; ces gneiss comprennent des bancs très siliceux de teinte bleutée, épais de 0,30 à $1 \mathrm{~m}$. On observe localement une alternance décimétrique de couches de gneiss et de filons de granite clair à grain fin; d'autres filons atteignent $5 \mathrm{~m}$ d'épaisseur. L'injection des filons de granite et de pegmatite a exercé une action mécanique sur les couches de gneiss résiduelles qui se trouvent soulevées et gondolées. Des plis tardifs, en tuiles, d'axe $\mathrm{N} 160^{\circ} \mathrm{E}$, déforment les bancs, ainsi que des ondulations en marches d'escalier, si communes dans tout le sud-est du Massif armoricain (Ters, 1979).

\section{Le contexte géographique}

La structure de pierres chauffées apparaissait en coupe de falaise, en retrait d'une tête de roche naturelle sur la

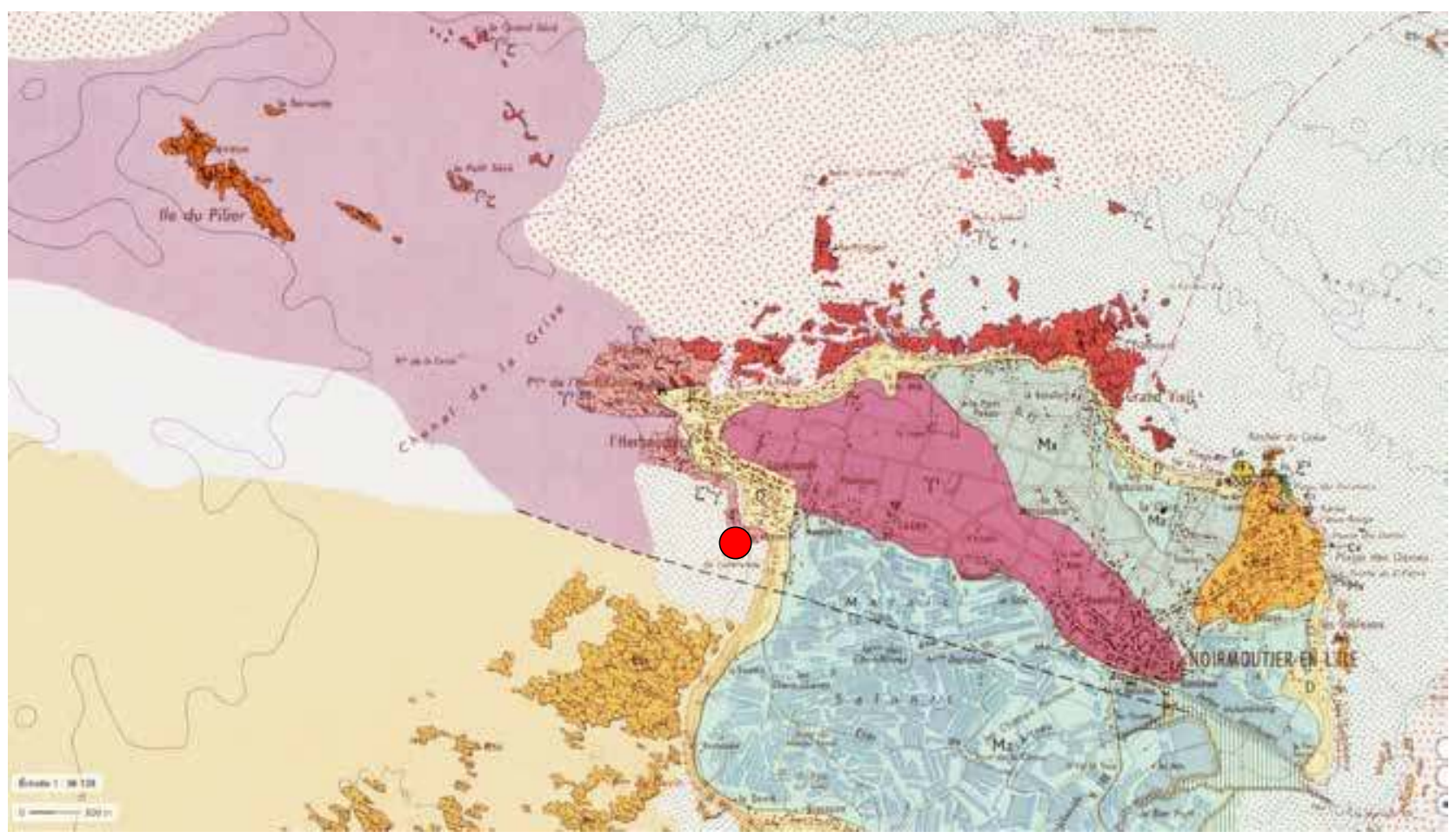

Figure 6 : Carte géologique du nord de l'île de Noirmoutier et situation du site (fonds BRGM).

Figure 6: Geological map of the north of the island of Noirmoutier and location of the site (BRGM background). 
falaise ouest de la pointe de Luzéronde, à quelque $20 \mathrm{~m}$ au nord-ouest de la fouille de F. Letterlé de 1986 qui, elle, était positionnée sur l'extrémité de la pointe. La tête de roche forme une protection relative de la falaise, ce qui a permis la sauvegarde partielle de la structure. De part et d'autre de ce rocher, on assiste actuellement au début de la formation de couloirs d'érosion marine qui entame le substrat, fragilisé par son réseau de diaclases. La dislocation de la falaise, là où la roche mère est fissurée, est en augmentation régulière et le phénomène s'est accéléré brutalement à partir du début des années 2010, année de la tempête Xynthia. Cette accélération de l'érosion est à surveiller dans ce secteur, notamment pour la préservation des indices d'occupations humaines mais aussi pour la conservation du milieu naturel. À l'occasion du relevé topographique lors de l'intervention d'octobre 2019, un relevé photogrammétrique a été réalisé comme référence afin d'évaluer par la suite l'érosion de la falaise dans ce secteur de la pointe de Luzéronde (fig. 7 et 8).

\section{Le contexte archéologique}

Le contexte archéologique du Néolithique dans l'île de Noirmoutier reste peu documenté et surtout peu précis. Pourtant nombreuses, les traces d'occupations néolithiques n'ont pas été relevées ou ont fait l'objet de descriptions sommaires et beaucoup de monuments mégalithiques ont été détruits. Les traces d'occupations domestiques et/ou artisanales sont quasiment inconnues, si ce n'est une présence attestée à Luzéronde, une autre sur l'île du Pilier (Large et al., 2013) et des " ateliers de taille » à l'anse Rouge et aux Champs-Pierreux.

Dans le contexte du pourtour de la baie de Bourgneuf, dont Noirmoutier forme la partie sud, les implantations néolithiques sont dominées par la présence d'un mégalithisme très important, connu grâce à des érudits locaux. Du moins l'était-il encore au XIX ${ }^{e}$ siècle car il a subi énormément de destructions depuis (fig. 9). À Noirmoutier, les mégalithes étaient situés sur la bande cristalline du nord de l'île, en bordure de la baie de Bourgneuf et des marais intérieurs de l'île. Quatre illustres chercheurs ont permis de se faire une idée de la densité de l'implantation des mégalithes dans cet étroit morceau de terre : Bachelot de La Pylaie (1832), Piet (1863), Charier-Fillon $(1888,1892)$ et Viaud-Grand-Marais (1884). Alors que sont visibles actuellement quatre monuments ${ }^{1}$, dont le dolmen de l'Herbaudière qui est en piteux état, ce ne sont pas moins de 24 monuments dont on a retrouvé la

1. Peut-être existe-t-il un cinquième et un sixième monument, encore visibles aujourd'hui. II nous a été signalé très récemment l'existence de vestiges mégalithiques dans une propriété du Bois de la Chaise. Ces restes méritent une évaluation avant de se prononcer sur leur authenticité. trace dans ces archives. On y rencontre des dolmens, des menhirs mais aussi une (ou plusieurs) file(s) de pierres dressées. Ces dernières sont signalées par F. Piet en 1864 à Saint-André. Il est possible d'inventorier 14 menhirs. F. Piet signale ceux de la Roche Levée, de la Roche à Payen, des Barres. De Bachelot de La Pylaie, nous retiendrons la Pierre Levée de Prélavé, toujours érigée dans une propriété, et celui du Bois de la Chaise, au phare, qui a été relevé en 1930. Enfin Charier-Fillon s'est attaché aux menhirs présents sur le plateau de la Vendette, menhirs qui étaient immergés à marée haute. Celui de la Table, longtemps pris pour un dolmen, est le seul actuellement à être encore visible (Large et al., 2015). Les autres ont servi de carrières de pierres : Riberge, Ténerge, La Truie. Deux autres menhirs sont encore signalés par cet auteur, l'un à l'ouest de l'île, Guérande, l'autre sur le plateau cristallin, les Charlières.

En ce qui concerne les dolmens, le dolmen de l'Herbaudière est encore visible bien que malmené par les assauts de la mer. Entre le 14 septembre et le 2 octobre 1987, une fouille a pu être réalisée dans le cadre d'une mission de sauvetage en raison de la destruction du monument par la mer (Poulain, 1988). Il avait été auparavant exploré en 1864 par J. Piet. Une partie du mégalithe gisait sur la plage, l'autre était enfouie sous la dune. Une cellule latérale du transept a pu être reconnue sous la dune ainsi que les restes d'un cairn (au NE). De ce transept, deux piliers étaient encore en place et les tables de couverture étaient effondrées. Le cairn est composé de moellons de granite sur au moins trois assises. Le mobilier comprend des tessons de céramique dont trois sont décorés au poinçon (Bronze ancien ou Campaniforme?), ainsi que de nombreux silex provenant de petits galets débités sur enclume. Tout ce mobilier était en position secondaire. D'autres dolmens de l'île ont disparu. Ils étaient déjà en fort mauvais état lors de leur reconnaissance au XIX ${ }^{e}$ siècle. Citons le dolmen de la Roche Groisard, repéré par Bachelot de La Pylaie, dont on ne connaît pas son plan. Celui du Chiron Tardiveau où deux chambres dolméniques étaient présentes. Il semble, d'après la description de J. Piet, que l'un des deux était un dolmen transepté. Le dolmen du Chiron Fassot était lui aussi en ruine lors de la visite de J. Piet, ainsi que ceux du Chiron de la Fée, de la Giraudière, des Pinaireaux, de la Roche à la Viaud, de la Roche au Breton, de la Roche Brûlée, tous signalés par J. Piet. Par intuition, J. Piet ajoute un dolmen probable à l'Échelle et un autre hypothétique dans le cimetière.

\section{La stratigraphie}

Comme le précisait F. Letterlé lors de son intervention de 1986, la stratigraphie mise en évidence est 


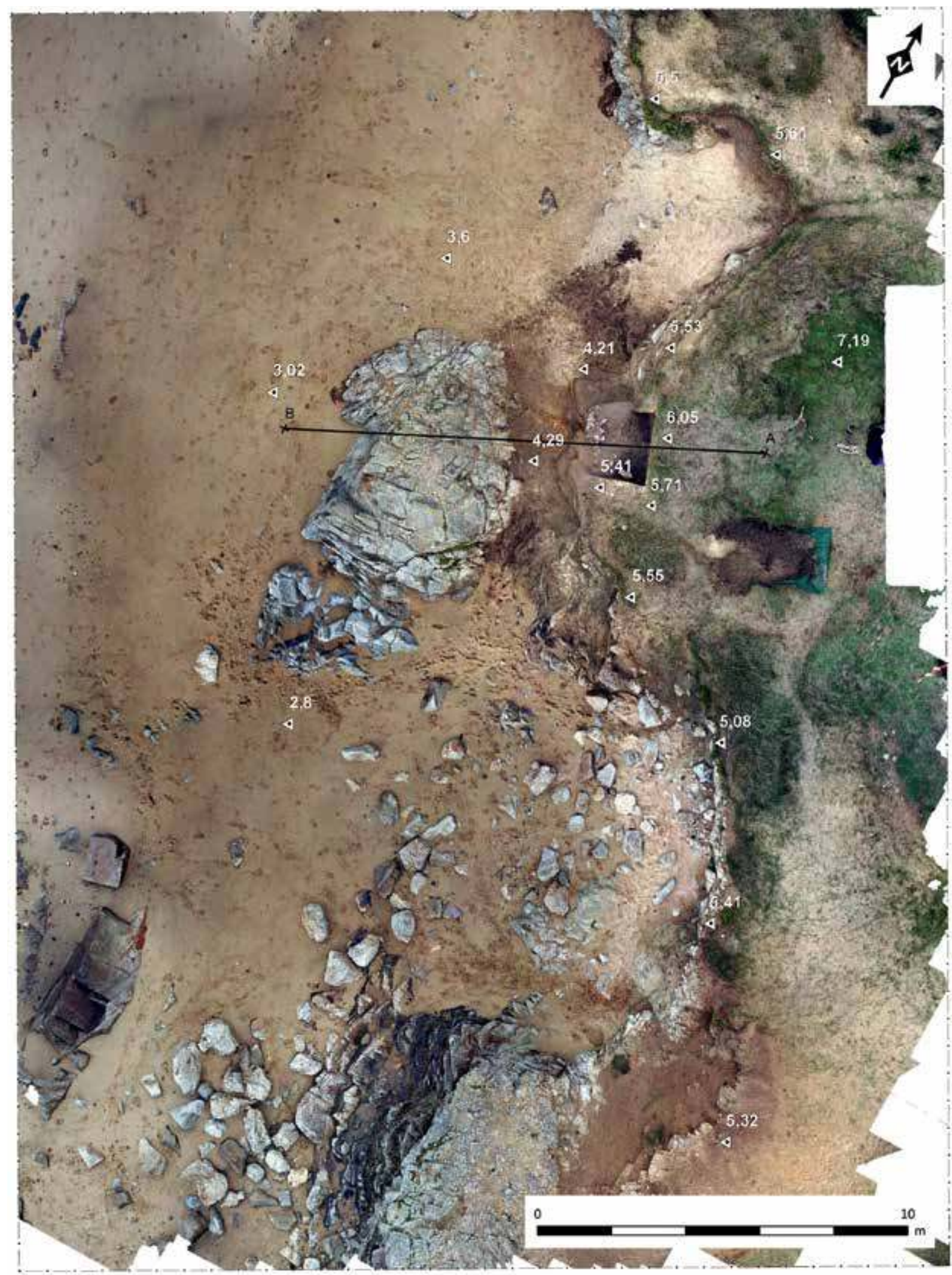

althudes rattachees su systeme de nivelement NGF-IGN69 intervalle des isohypses : $0,10 \mathrm{~m}$

Figure 7 : Orthophoto planaire (2 mm de résolution). L'intervention archéologique est située sur le tiers haut de la photo. Une section topographique la coupe en son milieu (réalisation T. Vigneau, 31/10/2019).

Figure 7: Planar orthophoto (2 $\mathrm{mm}$ resolution). The archaeological intervention is located in the upper third of the photo. A topographic section runs through the middle (produced by T. Vigneau, 31/10/2019). 


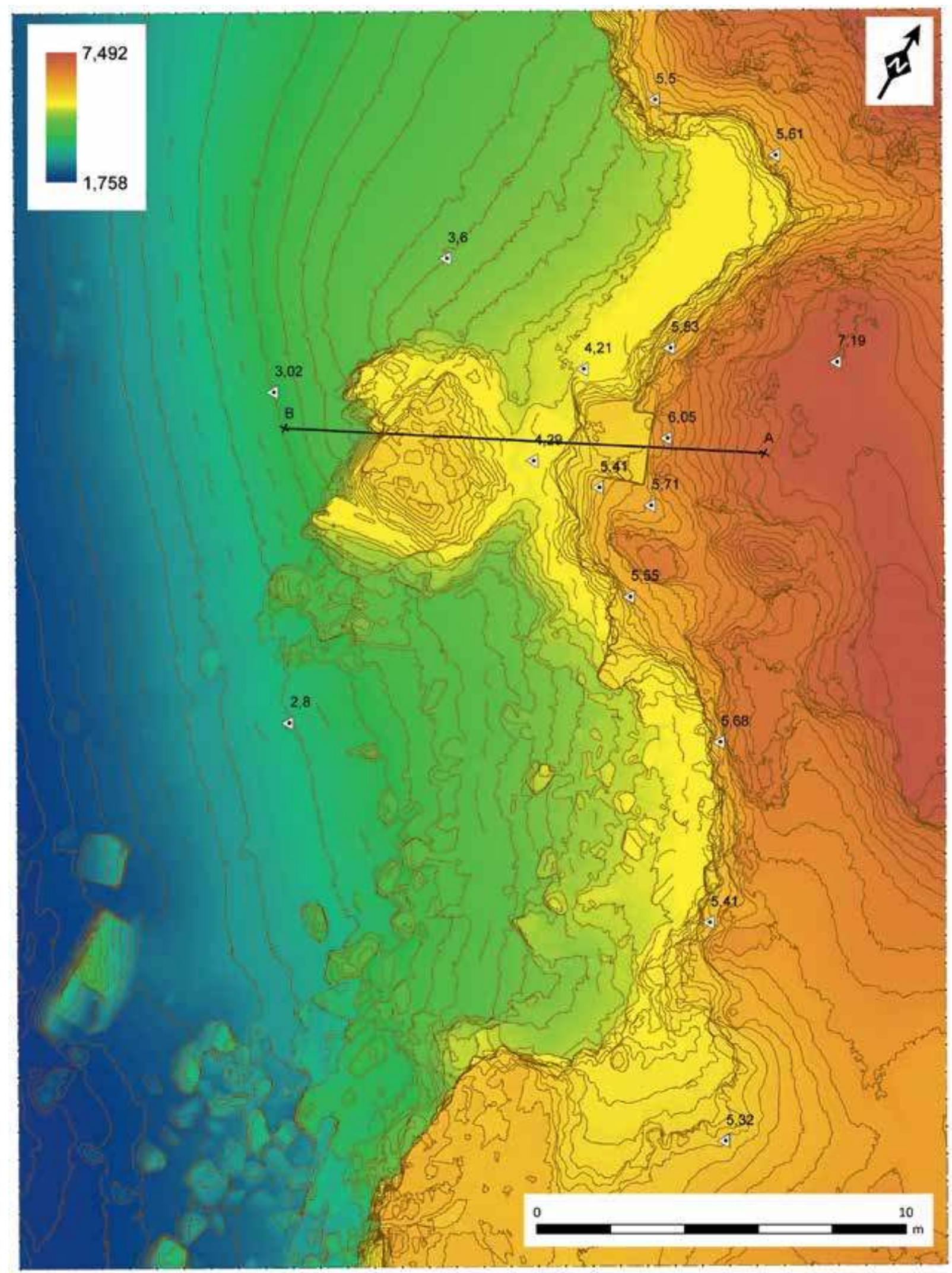

alteudes rattachees au systeme de nivelement NGF-IGNB9 intervalle des isolnypses : $0,10 \mathrm{~m}$

Figure 8: Modèle numérique de terrain (5 $\mathrm{mm}$ de résolution) [réalisation T. Vigneau, 31/10/2019]. 


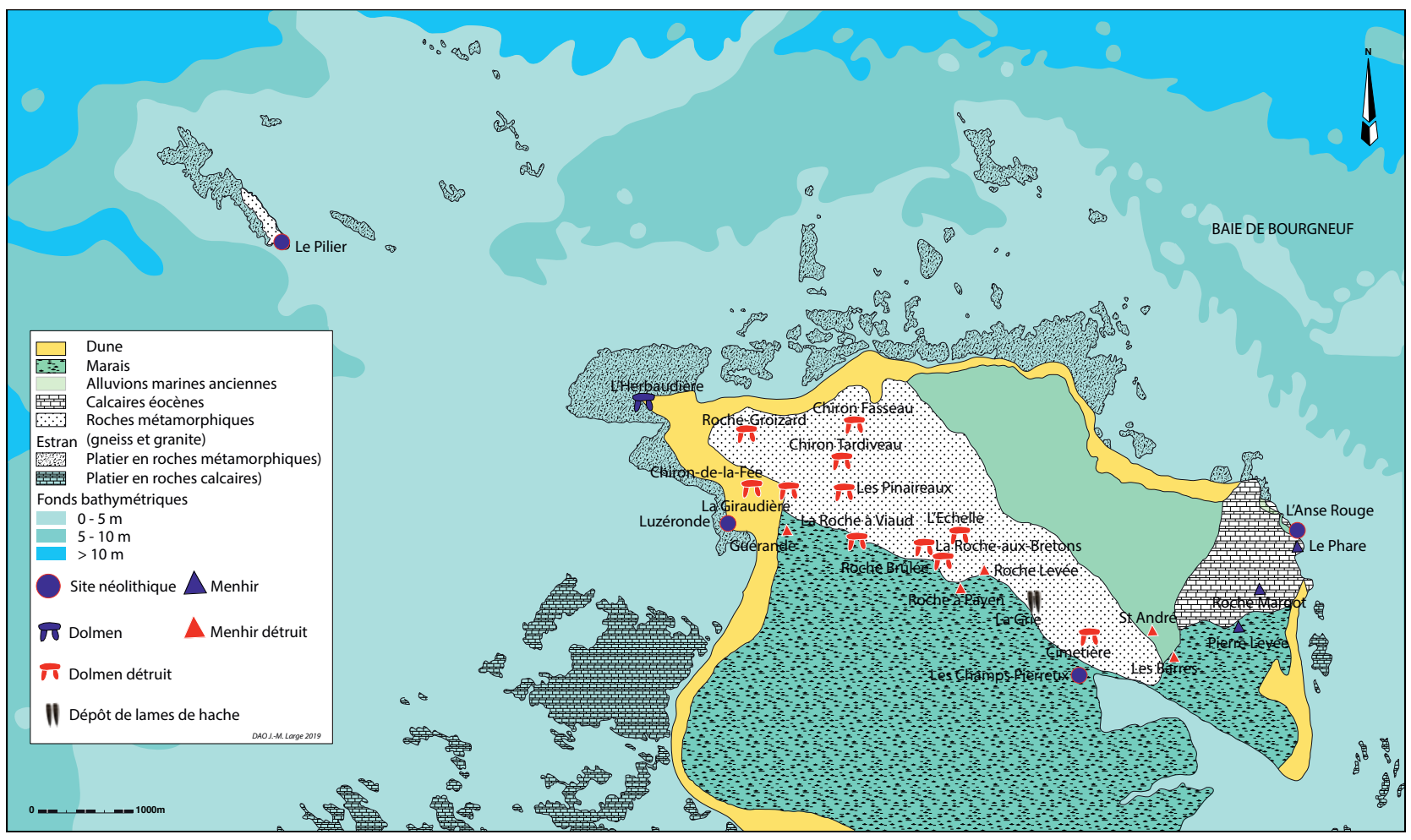

Figure 9: Carte des sites néolithiques au nord de l'île de Noirmoutier. Les sites domestiques et/ou artisanaux sont indiqués par un point. Les mégalithes sont indiqués par un signe distinctif (DAO J.-M. Large).

Figure 9: Map of Neolithic sites in the north of the island of Noirmoutier. Domestic and/or artisanal sites are indicated by a dot. Megaliths are indicated by a distinctive sign (CAD J.-M. Large).

simple. De son point de vue, la couche sablo-limoneuse, située sous la dune (US1), repose sur la roche (fig. 4). Ce n'est pas tout à fait ce qui a été mis en évidence lors de l'intervention d'octobre 2019. Il faut en effet distinguer sous la dune deux niveaux bien distincts de sables limoneux (fig. 10). Le premier (US2) a la particularité d'avoir une matrice très sableuse et une couleur qui passe du gris au gris sombre, au fur et à mesure que l'on descend dans la stratigraphie. Ce changement de couleur est dû à la migration des particules limoneuses qui s'enfoncent progressivement par illuviation pour atteindre un plancher induré, compact, celui du contact avec l'US3. Cette situation est très classique dans les comblements sablolimoneux que l'on rencontre sur l'ensemble du littoral en Vendée et en Bretagne. Ici, la couche US2 correspond à un niveau ayant subi anciennement un remaniement. Ce remaniement est bien visible par le mobilier qui a été recueilli : la plupart des tessons de céramique sont roulés et les plus récents semblent être datés du Bronze ancien, époque qui correspond sans doute à la mise en place progressive du réseau dunaire dans ce secteur de côte. La couche US3, dont le sommet forme le sol d'occupation initiale a une fraction argileuse plus marquée et, surtout, contient de nombreux fragments de roche issus du substrat gneissique à passées de pegmatite. Les quartz ont sou- vent des impacts éoliens, preuve de la mise en place de ce niveau en contexte périglaciaire. Le substrat de gneiss anatexique est très irrégulier, traversé par de nombreux filons de pegmatite et des masses de granite.

\section{Les faits archéologiques}

Les sept premières passes n'ont livré aucune structure perceptible. Ce sont les passes 8 et 9 qui ont permis de mettre en évidence deux faits archéologiques en place sur cette petite surface fouillée. La première correspond à l'ensemble cohérent de pierres chauffées qui était visible en coupe de falaise, la seconde est un gros bloc de gneiss autour duquel du mobilier archéologique a été retrouvé.

\section{La structure de pierres chauffées}

Composée presque exclusivement de blocs de petite taille $(<20 \mathrm{~cm})$ en gneiss, elle se présente en un seul niveau sur un sol limono-sableux compact (fig. 11). Il s'agit donc d'une structure à plat simple. Malgré la chute de certains blocs suite à l'érosion marine, la vingtaine de pierres subsistantes sont jointives et forment un ensemble homogène d' $1 \mathrm{~m}$ de large qui devait, à l'origine, être quasiment circulaire. La taille de cette structure correspond à celle de la plus grande 


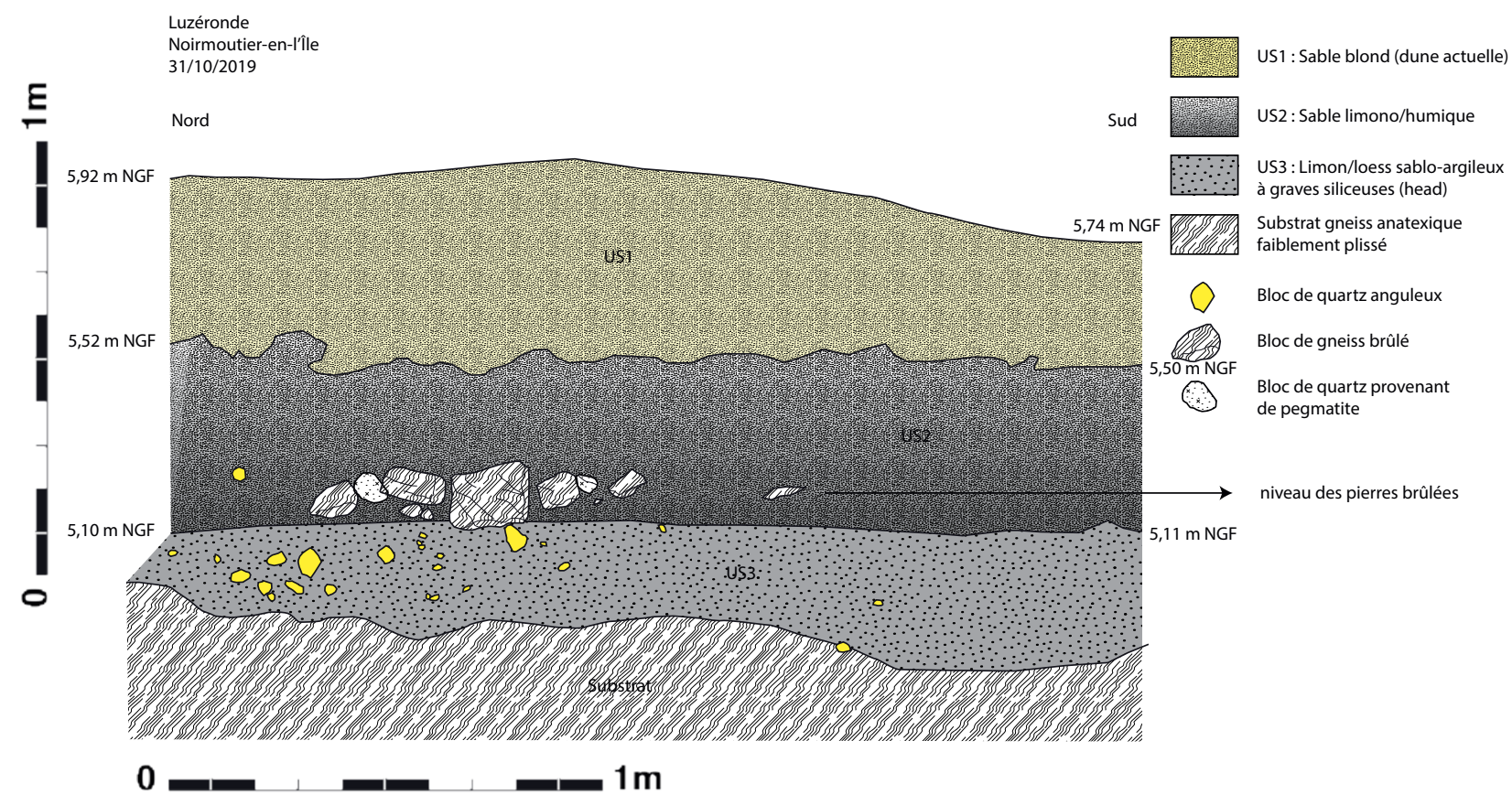

Figure 10 : Coupe nord-sud de l'intervention archéologique de 2019 (DAO J.M. Large).

Figure 10: North-south section of the 2019 archaeological intervention (CAD J.-M. Large).

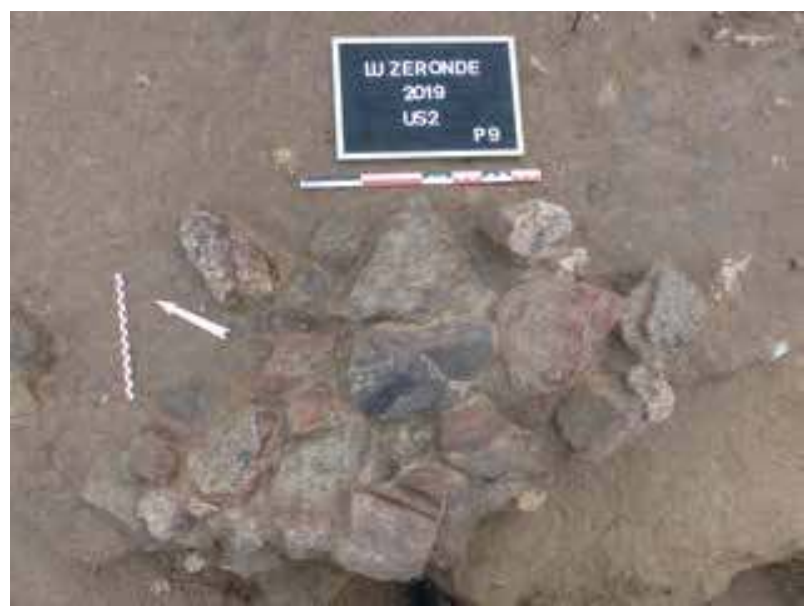

Figure 11: Cliché de la structure à pierres chauffées et plan des blocs perçus lors des passes 8 et 9 . On remarque le gros bloc de gneiss isolé au sud-est de la structure (cliché et DAO J.M. Large).

Figure 11: Photograph of the heated stone feature and plan of the blocks as observed during passes 8 and 9 . Note the large isolated gneiss block to the south-east of the feature (photo and CAD J.-M. Large).

partie des structures du même type connues dans l'ouest de la France au Néolithique (Lejay, 2011). Si les pierres présentent des traces de combustion, peu de charbons de bois ont pu être recueillis. Cette quasi-absence de charbons est probablement due à un lessivage. Quelques blocs de quartz (galets ou bloc issus de pegmatite) s'ajoutent aux moellons et galets de gneiss. Il faut noter l'abondance de silex brûlés

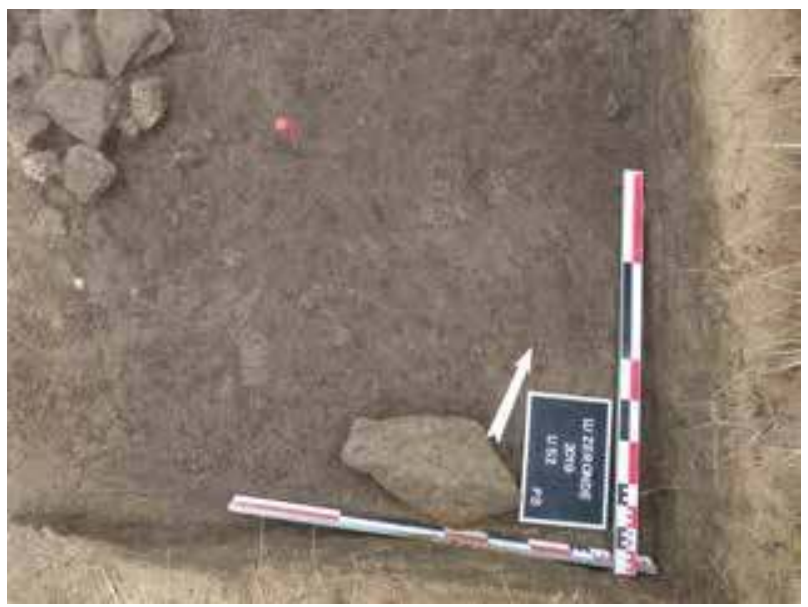

Figure 12 : Bloc de gneiss isolé (cliché J.M. Large).

Figure 12: Isolated gneiss block (photo J.M. Large).

puisse faire un lien technique entre le foyer et le débitage du silex. La technique du débitage par percussion directe n'a jamais été en lien avec une technique de taille par chauffage du silex, réservée plutôt à la technique de débitage lamino-lamellaire par pression, complètement absente ici.

\section{Le bloc de gneiss}

À $1,30 \mathrm{~m}$ à l'est de la structure à pierres chauffées, un gros bloc de gneiss $(40 \times 25 \times 15 \mathrm{~cm})$ est posé sur le même sol que la structure à pierres chauffées (fig. 12). Il repose 
à plat et, à son contact nord, un gros percuteur réalisé sur un galet éolisé de quartz, accompagne le bloc. Plusieurs percuteurs en galets de quartz éolisé, en bloc de quartz et en cristal de roche ont été retrouvés dans ce même niveau à proximité. Il s'agit là d'un fait intéressant dans la mesure où tous les éléments structuraux découverts étaient parfaitement en place, ce qui implique un enfouissement rapide, sans doute par du sable qui s'est fortement chargé en matière humique par la suite.

\section{Le mobilier}

Composé quasi exclusivement d'éléments lithiques et de tessons de céramique (seuls 3 fragments de coquillage ont été trouvés sur le sommet de la couche US2), le mobilier est relativement abondant. Au total, sur les $4 \mathrm{~m}^{2}$ fouillés dans l'US2, 176 éléments lithiques et 99 tessons ont été recueilli, soit 44 éléments lithiques au mètre carré et 24,75 tessons au mètre carré (fig. 13), à comparer avec ce que F. Letterlé avait trouvé en 1986 sur $10 \mathrm{~m}^{2}$ (189,2 éléments lithiques au mètre carré et environ 50 tessons au mètre carré). Si l'on observe ces densités, on s'aperçoit qu'elle décroit quand on se dirige le plus possible à l'ouest, au contact avec le bord de la falaise, ce qui sous-entend que la majorité du mobilier doit se trouver sous la dune actuelle, avec des niveaux archéologiques malheureusement très bouleversés par les implantations de défense allemandes.

\section{Le mobilier lithique}

Ce sont les éclats issus de galets côtiers qui sont majoritaires avec, aussi, une remarquable utilisation du quartz hyalin comme support d'éclats. Il provient du démantèlement de filons de pegmatite fréquents sur le site. Aucun support laminaire n'a été retrouvé, ce qui montre bien l'utilisation exclusive d'éclats avec ce type de support.

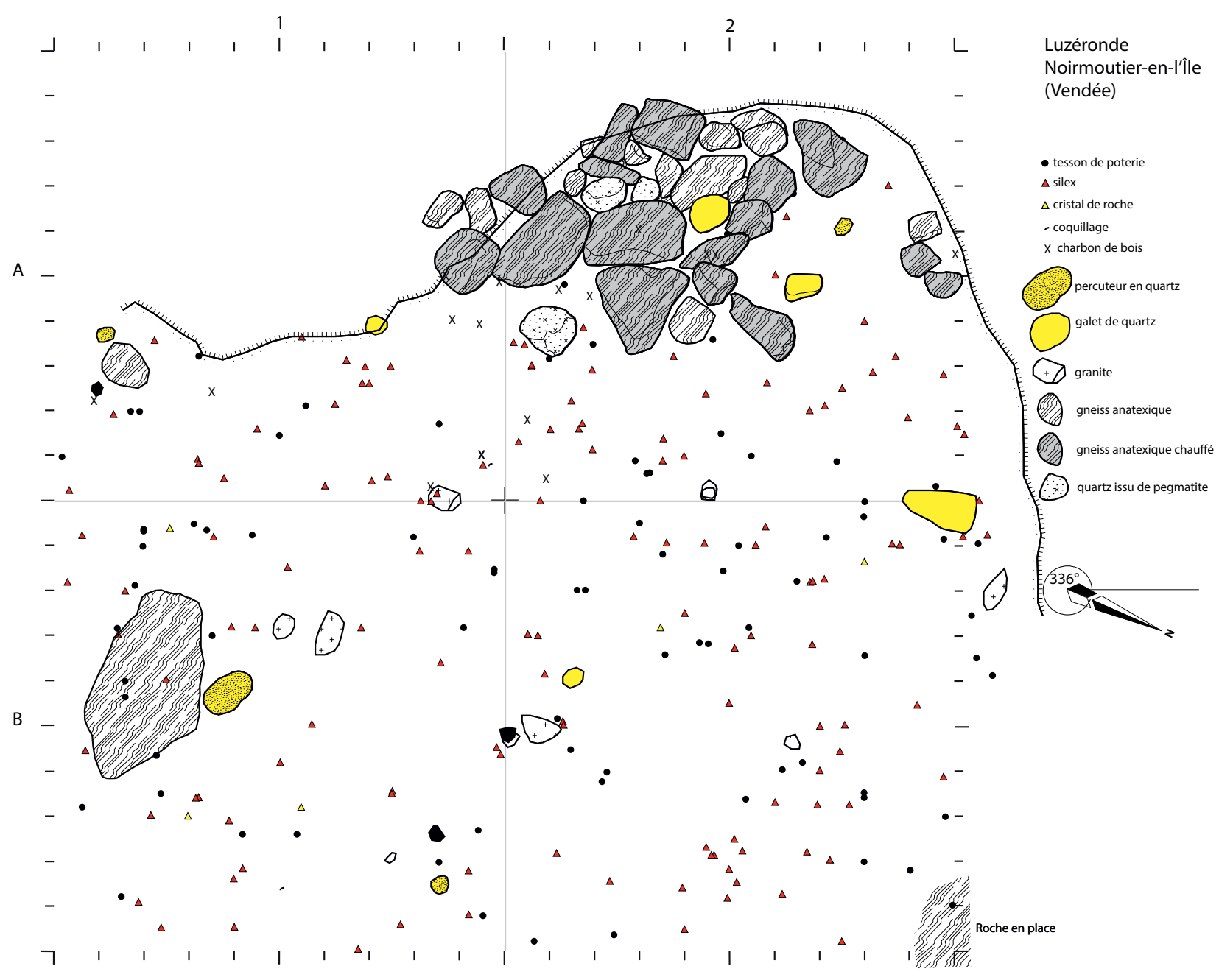

Figure 13 : Plan cumulatif de la dispersion du mobilier archéologique. Les dix passes de fouille de l'US2 sont représentées ensemble (DA0 J.-M. Large).

Figure 13: Cumulative plan of the dispersion of the archaeological artefacts. The ten excavation passes of US2 are represented together (CAD J.-M. Large). 


\begin{tabular}{|c|c|c|}
\hline $\begin{array}{c}\text { Silex issus } \\
\text { de galets }\end{array}$ & Quartz hyalin & Total \\
\hline 149 & 27 & 176 \\
\hline $84,66 \%$ & $15,34 \%$ & \\
\hline
\end{tabular}

Parmi les éclats, la présence de cortex montre bien l'utilisation de galets côtiers pour ce qui est du silex. Le débitage du silex est effectué par percussion directe dure, le débitage bipolaire sur enclume est anecdotique, un seul éclat présente ces stigmates (Guyodo et Marchand, 2005; Donnart et al., 2009). Les éclats retrouvés montrent bien une utilisation jusqu'au noyau du silex, ce qui est perceptible aussi sur les nucléus.

\begin{tabular}{|c|c|c|c|c|c|}
\hline $\begin{array}{c}\text { Éclat } \\
\text { très } \\
\text { cortical }\end{array}$ & Calotte & $\begin{array}{c}\text { Éclat } \\
\text { peu } \\
\text { cortical }\end{array}$ & $\begin{array}{c}\text { Éclat } \\
\text { moyen- } \\
\text { nement } \\
\text { cortical }\end{array}$ & $\begin{array}{c}\text { Éclat } \\
\text { non } \\
\text { cortical }\end{array}$ & Total \\
\hline 8 & 7 & 34 & 16 & 30 & 95 \\
\hline $8,42 \%$ & $7,37 \%$ & $35,79 \%$ & $16,84 \%$ & $31,58 \%$ & \\
\hline
\end{tabular}

\begin{tabular}{|c|c|c|c|}
\hline $\begin{array}{c}\text { Percussion sur } \\
\text { enclume }\end{array}$ & $\begin{array}{c}\text { Percussion } \\
\text { directe dure }\end{array}$ & Indéterminé & Total \\
\hline 1 & 71 & 23 & 95 \\
\hline $1,05 \%$ & $74,74 \%$ & $24,21 \%$ & \\
\hline
\end{tabular}

Quelques éclats accidents ont été retrouvés parmi l'ensemble du mobilier lithique.

\begin{tabular}{|c|c|c|c|c|}
\hline Réfléchi & Outrepassé & Siret & Torse & Total \\
\hline 11 & 0 & 6 & 0 & 17 \\
\hline
\end{tabular}

Les nucléus, certes peu nombreux mais bien présents, indiquent une utilisation active sur place des matériaux, ce qui est confirmé par la présence de percuteurs. À noter que l'un des nucléus est en quartz hyalin légèrement fumé.

\begin{tabular}{|c|c|c|c|c|}
\hline $\begin{array}{c}\text { Nucléus } \\
\text { unipolaire }\end{array}$ & $\begin{array}{c}\text { Nucléus } \\
\text { bipolaire }\end{array}$ & $\begin{array}{c}\text { Nucléus à } \\
\text { plans de frappe } \\
\text { orthogonaux }\end{array}$ & $\begin{array}{c}\text { Nucléus } \\
\text { multipolaire }\end{array}$ & Total \\
\hline 3 & 1 & 0 & 3 & 7 \\
\hline
\end{tabular}

\begin{tabular}{|c|c|c|}
\hline Nucléus à éclats & Nucléus à lames & Total \\
\hline 7 & 0 & 7 \\
\hline
\end{tabular}

Aucun élément laminaire n’a été retrouvé. 14 esquilles s'ajoutent à cet inventaire. Ce qui semble notable, c'est l'importance des silex qui ont subi une action thermique. Sur les 149 silex retrouvés, 42 présentaient des stigmates nets de chauffe $(28,19 \%)$, ce qui est à mettre en rapport avec la présence des pierres chauffées dans l'espace de fouille. Il s'agit très probablement de présence accidentelle d'éclats de silex dans le foyer.

L'outillage retrouvé est très faible (7 restes d'outils sur 149 silex, soit $4,70 \%): 2$ grattoirs sur éclats corticaux et un denticulé taillé à cœur; 2 fragments de lamelles; une pièce esquillée avec présence des esquilles sur deux bords opposés; un probable fragment de microlithe (bruit de fond mésolithique, comme l'avait noté F. Letterlé en 1986?) [fig. 14, nos 1 à 5].

Le macro-outillage est bien plus intéressant (fig. 14 et 15). À proximité du gros bloc de gneiss situé à l'est de la zone à pierres chauffées, un beau percuteur en galet de quartz éolisé présente des stigmates de percussion sur les deux faces opposées les plus petites. Ce bloc, très régulier (parallélépipédique), est tout à fait remarquable (fig. 14, $\left.\mathrm{n}^{\circ} 10\right)$ et d'autres percuteurs s'ajoutent à cet objet (5 en tout, présents uniquement dans la passe 8 , c'est-à-dire au contact du sol ancien, celui qui supporte les pierres chauffées). Les autres percuteurs sont aussi réalisés en galets de quartz éolisé dont certains sont brisés (fig. 14, n 9). L'un est un bloc de quartz issu d'un galet : il présente un bri à sa partie médiane : l'objet a été cassé en plusieurs morceaux (fig. $15, n^{\circ} 1$ ). Un petit percuteur est en quartz hyalin (fig. 14, $n^{\circ}$ ).

La présence de ce macro-outillage spécifique ainsi que l'abondance relative d'éclats en silex et en quartz plaident pour la présence en cet endroit d'un atelier de débitage dont les traces n'ont pas été modifiées : tout semble être resté figé depuis l'époque de l'utilisation fonctionnelle des lieux contrairement aux observations effectuées par F. Letterlé sur une partie du site située à une quinzaine de mètres plus au sud. Les traces de cette activité ont semblet-il été rapidement enfouies par un sable très humique, ce qui a permis la préservation des structures. Cela engage d'autant plus à surveiller l'érosion de ce secteur de l'île : d'autres structures peuvent se retrouver encore intactes malgré la modification des lieux par la marine allemande.

\section{La céramique}

Les tessons de céramique forment les autres indices chronoculturels présents sur l'espace de la fouille. 99 tessons ont été retrouvés et ils sont tous issus de céramiques modelées. Leur grand morcellement et l'état des cassures et des surfaces indiquent qu'ils ont été roulés. Peu d'éléments diagnostiques ont été retrouvés alors que la céramique reste un indicateur important pour déterminer les contextes chronoculturels. 

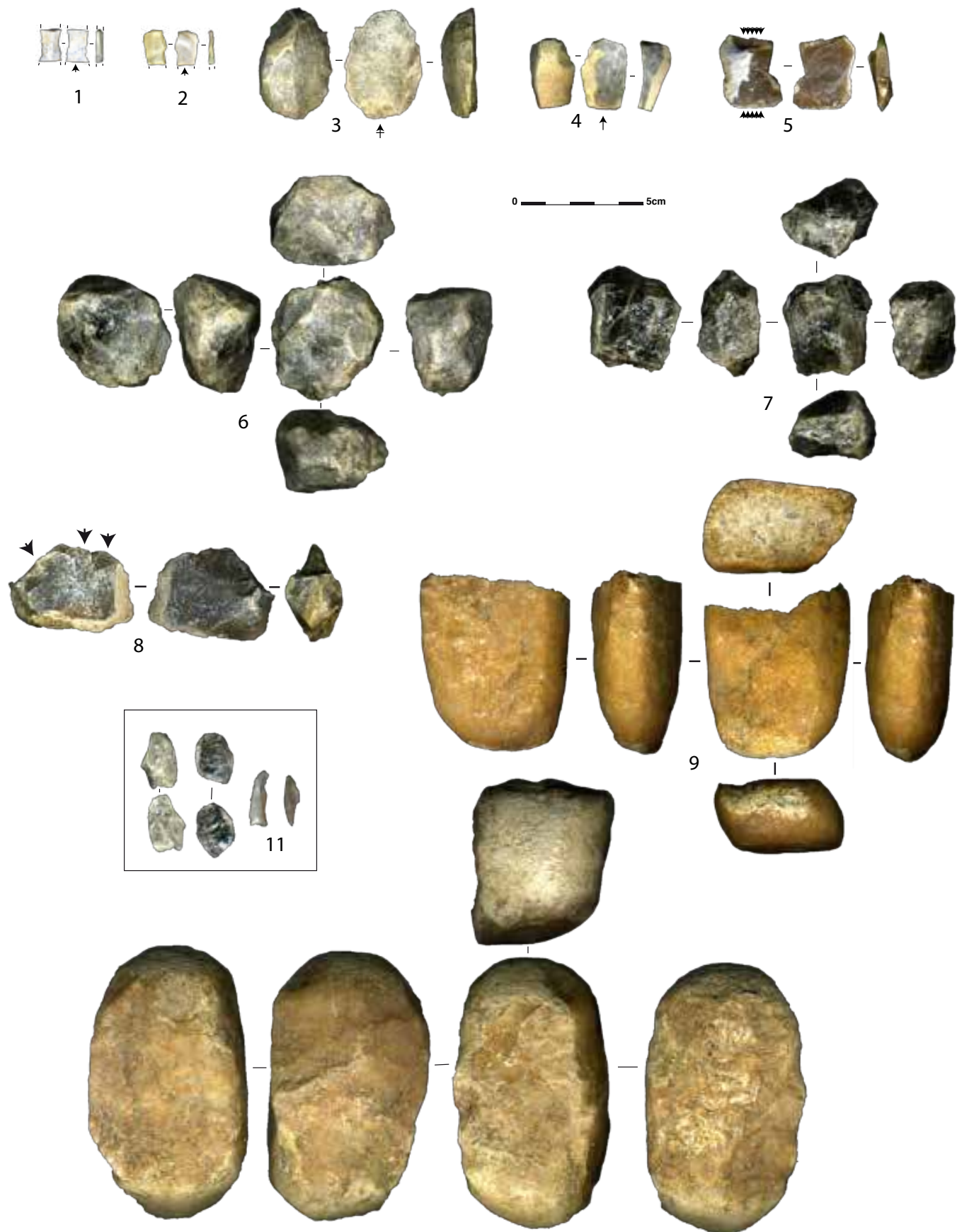

10
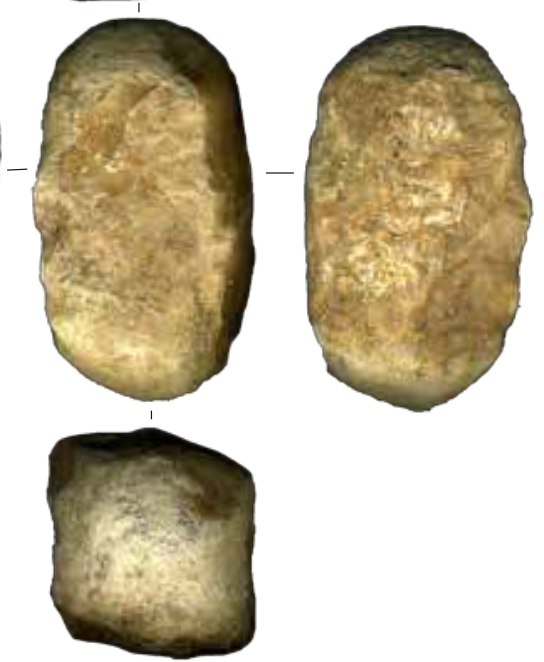

Figure 14 : Mobilier lithique.

Figure 14: Lithic material.

1-2: lamelles ; 3 : denticulé ; 4 : grattoir ; 5 : pièce esquillée ; 6 : percuteur en quartz; 7 : percuteur en galet de quartz éolisé ; 8 : nucléus unipolaire en silex; 9 : percuteur brisé en galet de quartz; 10 : gros percuteur en galet de quartz éolisé, découvert près du bloc de gneiss isolé ; 11 : dans l'encadré noir, éclat de quartz hyalin (DAO J.-M. Large).

1-2: flakes; 3: denticulates; 4: scraper; 5: chipped piece; 6: quartz striker; 7: eolian quartz pebble striker; 8: single-pole flint nuclease; 9: broken quartz pebble striker; 10: large eolian quartz pebble striker, found near the isolated gneiss block; 11: in the black box, hyaline quartz chip (CAD J.-M. Large). 


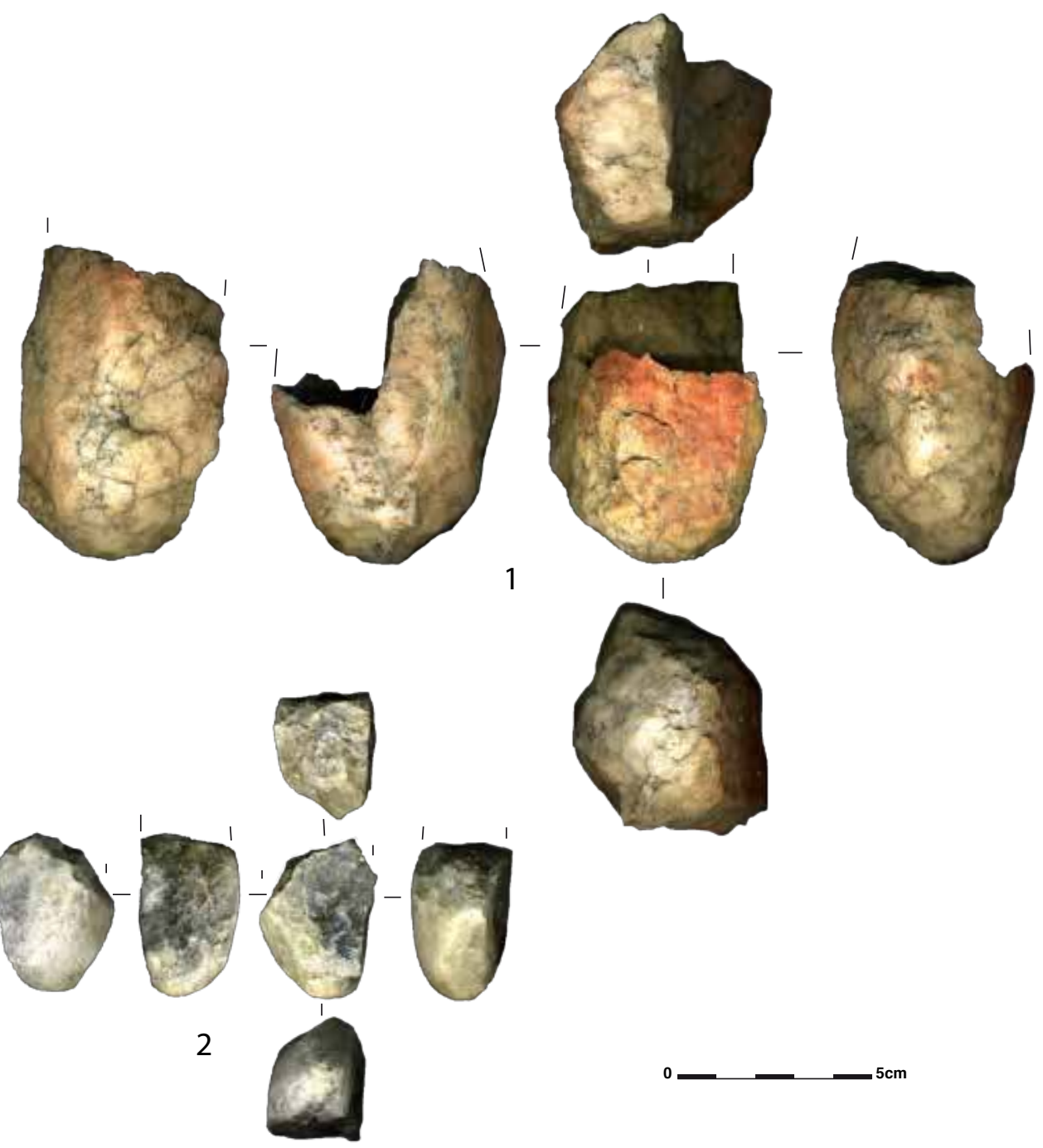

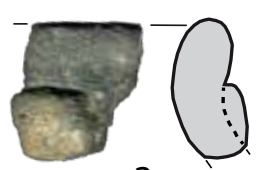

3

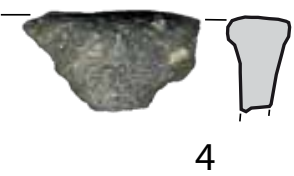

4
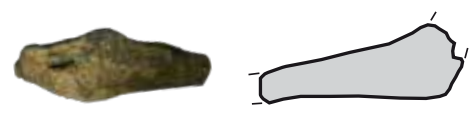

8

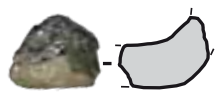

5

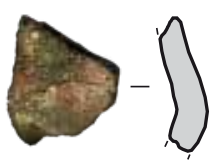

6

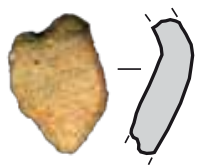

9

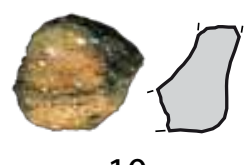

10

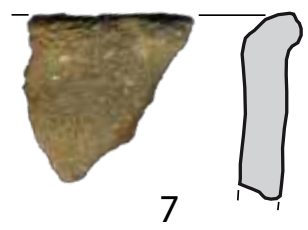

7

Figure 15 : Mobilier lithique et céramique

Figure 15: Lithic and ceramic material.

1 : percuteur brisé ; 2 : percuteur en quartz hyalin ; 3-10 : tessons de poterie (DA0 J.-M. Large). 


\begin{tabular}{|c|c|c|}
\hline Longueur & Largeur & Épaisseur \\
\hline $2,25 \mathrm{~cm}$ & $1,70 \mathrm{~cm}$ & $0,74 \mathrm{~cm}$ \\
\hline
\end{tabular}

Taille moyenne des tessons

La taille des tessons est très faible (inférieure à $4 \mathrm{~cm}^{2}$ ). Peu de tessons sont donc d'une taille suffisante pour permettre une analyse précise. Leur épaisseur est aussi peu importante, ce qui laisse de côté des vases de stockage. Ce sont surtout des restes de vases de cuisson et à boire. La nature de la pâte est quasiment la même pour tous les tessons rencontrés : il s'agit d'une pâte moyennement cuite à inclusions de quartz et de mica, ce qui dénote des tessons NACA trouvés à proximité du site qui présentaient une nature de pâte bien différente avec la présence, notamment, d'argilite ou de chamotte. Les inclusions sont souvent fines mais certaines peuvent atteindre plusieurs millimètres, correspondant à un tamisage peu soigneux de l'argile. Le degré de finition est lui aussi médiocre, le brunissage est présent sur deux tessons seulement, pour les autres il s'agit d'un lissage moyen.

\begin{tabular}{|c|c|c|}
\hline Lissage & Brunissage & Indéterminé \\
\hline 55 & 2 & 42 \\
\hline
\end{tabular}

Paroi externe

\begin{tabular}{|c|c|c|}
\hline Lissage & Brunissage & Indéterminé \\
\hline 63 & 0 & 36 \\
\hline
\end{tabular}

Paroi interne

\begin{tabular}{|c|c|}
\hline Érodées & Franches \\
\hline 91 & 8 \\
\hline
\end{tabular}

Cassures

Les éléments diagnostiques ne sont pas présents dans les passes au contact avec le sol ancien (passes 8 à 10). 3 éléments de probables fonds plats montrent bien une phase récente de leur fabrication (Néolithique récent à Bronze moyen). Ils sont présents dans la partie sommitale de l'US2, là où le remaniement a été le plus important. Peu de bords ont été repérés, seul un tesson présente un bord éversé avec la présence d'un pastillage ou d'un fragment de cordon. Il a été trouvé tout au sommet de l'US2, ce qui ne plaide pas pour une relation directe avec la structure à pierres chauffées. Trois tessons présentent une rupture de pente, signalant des vases à paroi segmentée, que l'on peut rapprocher d'un tesson Groh-Collé trouvé lors des prospections pédestres (fig. 15, $\mathrm{n}^{\circ} 6$ ).

Qu'ils soient lithiques ou céramiques, les artéfacts ne livrent pas particulièrement une indication chronocultu- relle précise : l'ensemble des éléments peut être compris du Néolithique récent jusqu'au Bronze moyen. Cela ne préjuge en rien de la datation de la structure à pierres chauffées qui n’a révélé aucun élément diagnostique précis, d'autant que la plupart des tessons présentent des cassures érodées : ils ont bougé dans les sédiments sablo-argileux.

\section{Prélèvement de charbons de bois et datation}

Heureusement, du fait de la présence de la structure à pierres chauffées, il a été possible de recueillir des échantillons de charbons de bois dans des conditions tout à fait satisfaisantes, sous les pierres brûlées, au contact avec le sol ancien. La datation de ces échantillons obtenue par accélérateur a livré une période d'utilisation de la structure de combustion entre 4486 et 4342 av. n. è. (95,4\% de probabilité - Lyon-17382 (GrM) : $5570 \pm 40 \mathrm{BP})$.

\section{BREF ESSAI DE CONTEXTUALISATION}

La présence de foyers dans les sites d'occupation du Néolithique moyen dans l'Ouest de la France est beaucoup mieux connue depuis le développement des fouilles préventives. Ceux-ci peuvent avoir différentes formes et typologies. Un travail récent (Lejay, 2011) a permis de les catégoriser afin de mieux maîtriser leur typologie et envisager leur fonctionnalité. Sept catégories de structures de combustion ont ainsi été identifiées :

- les structures à plat simples;

- les structures à plat limitées;

- les structures à plat à pierres chauffées;

- les structures en creux simples;

- les structures en creux limitées;

- les structures en creux à pierres chauffées;

- les structures architecturées.

L'ensemble de ces structures se retrouvent souvent combinées sur un même site d'occupation du $\mathrm{V}^{\mathrm{e}}$ millénaire.

La structure de Luzéronde appartient à la catégorie des structures à plat à pierres chauffées que l'on retrouve sur plusieurs sites régionaux (Bellanger, 2010; Hinguant, 2010; Laporte et al., 2003; Large et Birocheau, 2004; Le Roux, 2006; Marchand et al., 2009; Poissonnier et al., 2006, etc.). Ces structures sont souvent de dimensions assez réduites, de l'ordre du mètre carré, et leur forme est variée, de circulaire à ovalaire, voire quadrangulaire. Dans la mesure où le foyer de Luzéronde a été tronqué par l'érosion marine, il est délicat de lui attribuer une forme précise.

La mise en place d'une structure de combustion à plat nécessite souvent la préparation de la zone où elle va être installée en nettoyant les abords et en aménageant un lit de pierres (ce qui n'est pas systématique) constitué 
de petits blocs et de galets à Luzéronde. La présence de galets indique que ces pierres ont été ramassées de manière opportune. La nature du combustible n'a pas été vérifiée à Luzéronde en raison de l'indigence des charbons de bois, plutôt réservés à l'obtention d'une datation au radiocarbone. Dans les études régionales réalisées sur ce type de foyers (Lejay, 2011), c'est l'utilisation du chêne qui domine avec les pomoïdés. Dans le cas des sites de bord de mer, d'autres essences peuvent être envisagées. Il est délicat d'entrevoir la fonction du foyer de Luzéronde tant les possibilités sont nombreuses : grillage, friture, bouilli, fumage, voire même éclairage d'une zone côtière, ce qui peut être dangereux pour la navigation par cabotage, point central d'activités sociales (la taille de silex à proximité est une activité reconnue à Luzéronde), chauffage de personnes... Toutes ces utilisations peuvent être envisagées pour le foyer de Luzéronde...

\section{CONCLUSION}

L'intervention sur la structure à pierres brûlées de la pointe de Luzéronde a permis de se rendre compte de la qualité des informations que l'on peut recueillir sur ce site, malgré les bouleversements qui ont eu lieu lors de l'aménagement du Mur de l'Atlantique. La côte ouest de la pointe, particulièrement susceptible de recevoir les coups de boutoir de la mer au moment des tempêtes hivernales, s'érode assez rapidement : la décennie actuelle est la période où la dynamique côtière est la plus visible. Érosion et accrétion sableuse sont les deux facteurs principaux de la modification du trait côtier auxquels s'ajoutent le piétinement par les visiteurs et la pression immobilière. La pointe de Luzéronde n'échappe évidemment pas à ces modifications sensibles, et le site archéologique encore mal défini actuellement, avec une période d'occupation couvrant trois millénaires, en subit les conséquences. Un suivi régulier a permis de déceler des informations nouvelles et d'intervenir très légèrement sur une partie du site qui montrait en coupe de falaise un potentiel intéressant.

Plusieurs tessons du Néolithique ancien avaient été trouvés dans ce secteur par prospections pédestres. Cela engageait une possibilité pour que la structure perçue en coupe ait une grande ancienneté. Si l'intervention de 2019 n'a pas permis de trouver des éléments diagnostiques pour confirmer ou infirmer cette hypothèse, une datation au radiocarbone positionne la structure de combustion dans le troisième quart du $\mathrm{V}^{\mathrm{e}}$ millénaire, c'est-à-dire au Néolithique moyen 1 . Associés à cette structure, des éléments de débitage lithique corroborent l'hypothèse de la présence d'un atelier de débitage à proximité. Il est possible que cette structure soit liée à une activité culinaire, sans que l'on puisse en apporter la preuve.

\section{Bibliographie}

Bachelot de La Pylaie J.-M., 1832, « Lettres de M. de La Pylaie sur les antiquités de Noirmoutier à M. Huette, membre de la Société académique et opticien à Nantes ", Annales de la Société royale académique de Nantes et du département de Loire-Inférieure, 3, p. 168-172.

Bellanger P. (dir.), 2010, Guérande, Loire-Atlantique, ZAC de Bréhadour, le parc Savari. Occupations du Néolithique moyen I et de la transition des deux âges $d u$ Fer, rapport final d'opération, Cesson-Sévigné, Inrap Grand-Ouest, 244 p.

Blanchet S., Forré P., Fromont N., Hamon C. et HaMON G., 2010, "Un habitat du Néolithique ancien à Betton " Pluvignon" (Ille-et-Vilaine) ", dans Billard C. et Legris M. (dir.), Premiers Néolithiques de l'Ouest, Rennes, Presses universitaires de Rennes, p. 15-39.

Charier-Fillon A., 1888, Lîle de Noirmoutier, II, Contribution à l'étude des mouvements du sol, Niort, Clouzot, libraire-éditeur, $17 \mathrm{p}$.

Charier-Fillon, 1892, Lîle de Noirmoutier, Péril et défense, Niort, Clouzot, libraire-éditeur.

Chazette A., 2010, Noirmoutier sous l'Occupation allemande. Maillon du Mur de l'Atlantique, Paris, Histoire et Fortifications, $64 \mathrm{p}$.

Donnart K., Naudinot N. et Le Clézio L., 2009, “ Approche expérimentale du débitage bipolaire sur enclume : caractérisation des produits et analyse des outils de production ", Bulletin de la Société prébistorique française, 106, 3, p. 517-533.

Dupont F., Liagre J. et Irribaria R., 2010, "Sours - Les Ouches (Eure-et-Loir) ", dans BILlard C. et Legris M. (dir.), Premiers Néolithiques de l'Ouest, Rennes, Presses universitaires de Rennes, p. 75-100.

Gachina J., Gomez J. et Joussaume R., 1991, "Néolithique ancien de la grotte de Bois-Bertaud) à Saint-Léger-en-Pons (Charente-Maritime) ", Bulletin de la Société préhistorique française, 88, 1, p. 26-31.

Guyodo J.-N. et Marchand G., 2005, « La percussion bipolaire sur enclume dans l'Ouest de la France de la fin du Paléolithique au Chalcolithique : une étude économique et sociale ", Bulletin de la Société préhistorique française, 102, 3, p. 539-549.

Hinguant S. (dir.), 2010, Les structures à pierres chauffées $d u$ Néolithique moyen de Montauban (Carnac, Morbihan) : feux domestiques, feux rituels?, rapport final d'opération, Rennes, SRA Bretagne.

Joussaume R., 1981, Le Néolithique de l'Aunis et du Poitou occidental dans son cadre atlantique, Travaux du Laboratoire d'Anthropologie - Préhistoire - Protohistoire et Quaternaire armoricain, Rennes, ER 27 du CNRS, 620 p.

LApORTE L., 2003, "Quelques éléments céramiques attribuables au Néolithique ancien et au Néolithique récent 
provenant du site de la Pierre-Saint-Louis à Geay (Charente-Maritime) ", Bulletin du Groupe Vendéen d'Études Préhistoriques, 39, p. 14-27.

Laporte L. et Pice C. (dir.), 2002, «Les occupations néolithiques du vallon des Ouchettes (Plassay, Charente-Maritime) ", Gallia Préhistoire, 44, p. 1-120.

Laporte L., Bernard V., Bizien-Jaglin C., Dietch M.-F., Guyodo J.-N., Hamon C., Lemoulin Q., Luquin A., Noslier A. et Quesnel L., 2003, Lillemer (Ille-et-Vilaine), rapport de fouille programmé et de prospection thématique, Rennes, SRA Bretagne.

Large J.-M., 2009, "Témoins d'occupation du Néolithique ancien au Mûrier de Luzéronde, Noirmoutier-en-l'Île (Vendée) ", Revue archéologique de l'Ouest, 26, p. 37-41.

Large J.-M. et Birocheau P. (dir.), 2004, Les Châtelliers du Vieil-Auzay (Vendée), une archéologie d'un site exceptionnel de la préhistoire récente, La Roche-sur-Yon, Groupe Vendéen d'Études Préhistoriques, 686 p.

Large J.-M., Corson S. et Tortuyaux J.-P., 2013, « Prospection archéologique à l'île du Pilier (Noirmoutier-en-l'Île, Vendée) ", Bulletin du Groupe Vendéen d'Études Préhistoriques, 49 , p. 27-40.

Large J.-M., Corson S. et Magne J., 2015, « Le mégalithe de la Table sur le plateau de la Vendette (Noirmoutier-en-l'Île, Vendée) ", Bulletin du Groupe Vendéen d'Études Préhistoriques, 51, p. 33-41.

LEJAY M., 2011, Les structures de combustion néolithiques dans l'Ouest de la France, mémoire de master 1 Archéologie et Histoire, Rennes, université de Haute Bretagne, 133 p.

Le Roux C.-T. (dir.), 2006, Monuments mégalithiques à Locmariaquer (Morbihan). Le long tumulus d'Er Grah dans son environnement, Paris, supplément XXXVIII à Gallia Préhistoire, $308 \mathrm{p}$.
Letterlé F., 1986, Rapport de la fouille de sauvetage de Luzéronde à Noirmoutier (Vendée) - Autorisation 86-07, Nantes, SRA des Pays de la Loire, 11 p.

LÉvêque F., Gouraud G. et BouIn F., 1997, “ Contribution à l'étude des occupations préhistoriques de la grotte de la Grande Roche de la Plématrie à Quinçay (Vienne) ", Bulletin du Groupe Vendéen d'Études Préhistoriques, 33, p. 5-8.

Marchand G., Mens E., Carrion Marco Y., Berthaud G., Kerdivel G., George E., Quesnel L. et Vissac C., 2009, "Creuser puis brûler : foyers et carrières néolithiques de Mazières-en-Mauges "le Chemin Creux" (Maine-etLoire) ", Bulletin de la Société préhistorique française, 106, 4, p. 735-759.

Patte E., 1971, "Quelques sépultures du Poitou, du Mésolithique au Bronze ancien ", Gallia Préhistoire, 14, p. 139-244.

Piet F. et Piet J., 1863, Recherches topographiques, statistiques et historiques sur lîle de Noirmoutier, Nantes, imprimerie Mellinet.

Poissonnier B., Dubillot X., Forré P., Marchand S. et Pont-Tricoire C., 2006, Beaufort-en-Vallée, Le Boulerot, rapport final d'opération, Cesson-Sévigné, Inrap GrandOuest, $332 \mathrm{p}$.

Poulain H., 1988, " Mégalithe de la pointe de l'Herbaudière », Bulletin municipal de Noirmoutier-en-l'̂̂le, 21, p. 38-40.

Riche C., Ravon E. et Bedault L., 2010, "Le site VilleneuveSaint-Germain d'Aubevoye "La Chartreuse" (Eure) ", dans Billard C. et Legris M. (dir.), Premiers Néolithiques de l'Ouest, Rennes, Presses universitaires de Rennes, p. 41-63.

Ters M., 1979, Notice explicative de la carte géologique de la France au 1/50 000 - Ile de Noirmoutier, Pointe de SaintGildas, X-24-25, Orléans, Éditions du BRGM, 5 p.

Viaud-Grand-Marais A., 1884, Guide du voyageur à Noirmoutier, Nantes, Imprimerie de l'Ouest, $134 \mathrm{p}$. 


\section{Eine mittelneolithische Feuerstelle bei Luzéronde (Noirmoutier-en-l'Île, Vendée)}

Durch die fortwährende Veränderung der Küstenlinie durch das Meer in Form von Abbrüchen und Erosion wurden einige durch Feuer veränderte Steinblöcke an der Steilküste bei Pointe de Luzéronde, Noirmoutier-en-Île, festgestellt. Die archäologische Fundstelle war bereits bekannt; eine erste archäologische Untersuchung fand hier bereits 1986 statt. Die Feuerstelle, die jetzt zum Vorschein kam, war bei der vorhergehenden archäologischen Untersuchung noch nicht erfasst worden. Angesichts der Bedrohung der Fundstelle durch völlige Zerstörung infolge des Küstenabbruchs, fand im Oktober 2019 eine eng begrenzte Untersuchung statt, die es erlaubte, diesen an einen großen, aus Gneis bestehenden Gesteinsblock anschließenden Befund näher zu dokumentieren. Eine Radiokarbondatierung erlaubt es, den Befund dem dritten Viertel des 5. Jahrtausends v. Chr. zuzuweisen.

Schlagwortindex: Pays de la Loire, Vendée, Neolithikum, Feuerstelle.

\section{Un hogar del Neolítico medio en Luzéronde (Noirmoutier-en-l'Île, Vendée)}

Mientras que el mar continúa erosionando el litoral, algunos bloques quemados aparecieron en el perfil de un acantilado en la punta de Luzéronde en Noirmoutier. El yacimiento ya se conocía y fue objeto de una intervención arqueológica en 1986. La estructura de combustión que apareció no formaba parte del sector de la antigua intervención. Frente a la amenaza de una destrucción definitiva, una intervención muy limitada tuvo lugar en octubre de 2019 y permitió acotar la estructura asociada a un gran bloque de gneis. Una datación radiocarbónica la sitúa en el tercer cuarto del $V$ milenio antes de nuestra era.

Palabras clave: Países del Loira, Vendée, Neolítico, hogar. 